\title{
osr1 maintains renal progenitors and regulates podocyte development by promoting wnt2ba through antagonism of hand 2
}

\author{
Bridgette E. Drummond ${ }^{1}$, Brooke E. Chambers ${ }^{1}$, Hannah M. Wesselman ${ }^{1}$, Marisa N. Ulrich ${ }^{1}$, \\ Gary F. Gerlach ${ }^{1}$, Paul T. Kroeger ${ }^{1}$, Ignaty Leshchiner ${ }^{2}$, Wolfram Goessling ${ }^{2}$, \\ and Rebecca A. Wingert ${ }^{1 *}$ \\ ${ }^{1}$ Department of Biological Sciences, Center for Stem Cells and Regenerative Medicine, Center \\ for Zebrafish Research, University of Notre Dame, Notre Dame, 46556, USA \\ ${ }^{2}$ Brigham and Women's Hospital, Genetics and Gastroenterology Division, Harvard Medical \\ School, Harvard Stem Cell Institute, Boston, MA 02215, USA \\ *Corresponding author
}

Keywords: zebrafish, kidney, nephron, podocyte, segmentation, osrl, wnt2ba, hand2

\begin{abstract}
Abbreviations: chronic kidney disease (CKD), N-ethyl-N-nitrosourea (ENU), days post fertilization (dpf), hours post fertilization (hpf), whole mount in situ hybridization (WISH), flourescent in situ hybridization (FISH), immunofluorescence (IF), immunocytochemistry (ICC), intermediate mesoderm (IM), congenital anomalies of the kidney and urinary tract (CAKUT), amino acid (aa), Zebrafish International Research Center (ZIRC), capped RNA (cRNA), somite stage (ss), wild-type (WT), LIM homeobox la (lhxla), paired box $2 a$ (pax2a), wilms tumor la (wt1a), wilms tumor $1 b$ (wt1b), nephrosis 1, congenital Finnish type (nephrin) (nphs1), nephrosis 2, idiopathic, steroid-resistant (podocin)(nphs2), odd-skipped related transcription factor 1 (osr1), cadherin 17 (cdh17), odd-skipped related transcription factor 2 (osr2), wingless-type MMTV integration site family, member $2 \mathrm{Ba}$ (wnt2ba) and wingless-type MMTV integration site family, member 2Bb (wnt2bb), heart and neural crest derivatives expressed 2 (hand2), myosin light chain (myl7).
\end{abstract}

Correspondence: Rebecca A. Wingert, Ph.D., Department of Biological Sciences, University of Notre Dame, 100 Galvin Life Sciences, Notre Dame, IN 46556, USA; Email: rwingert@nd.edu, Phone: (574)-631-0907, Fax: (574)-631-7413 


\begin{abstract}
Knowledge about the genetic pathways that control renal cell lineage development is essential to better understand the basis of congenital malformations of the kidney and design regenerative medicine therapies. The embryonic zebrafish kidney, or pronephros, contains two nephrons that are conserved with humans. Recently, the transcription factors Osr1 and Hand2 were found to exert antagonistic influences to balance kidney specification (Perens et al., 2016). Here, we performed a forward genetic screen in zebrafish to identify nephrogenesis regulators, where whole genome sequencing of the novel oceanside (och) mutant revealed a nonsense mutation in osr 1 . ocn mutants evince severe pronephros defects including abrogation of podocytes and proximal tubule cells. Our studies reveal that osrl is not needed to specify renal progenitors, but rather required to maintain their survival. Additionally, osrl is requisite for expression of the canonical Wnt ligand wnt2ba, where wnt2ba is expressed in the intermediate mesoderm (IM) and later restricts to podocytes. Deficiency of $w n t 2 b a$ reduced podocyte progenitors, where overexpression of wnt2ba was sufficient to rescue the podocyte lineage as well as osrl loss of function. Finally, we demonstrate that reciprocal antagonism between osrl and hand2 mediates podocyte development specifically by controlling wnt $2 b a$ expression in the IM. Together, our data show that Osr1 is essential for a sequence of temporal functions that mediate the survival and lineage decisions of IM progenitors, and subsequently the maintenance of podocytes and proximal tubule epithelium in the embryonic nephron.
\end{abstract}




\section{INTRODUCTION}

The kidney is the organ that cleanses our blood and initiates the process of waste excretion. The portion of the kidney that makes this possible are the nephrons, which are composed of a blood filter, tubule and collecting duct. The blood filter itself is composed of several cellular features including a capillary bed with a fenestrated endothelium, known as the glomerulus, and the space that the glomerulus is housed in, known as the Bowman's capsule. Octopus-like epithelial cells known as podocytes are situated in opposition to a specialized glomerular basement membrane (GBM) surrounding the capillaries (Ichimura et al. 2017). Filtration is accomplished due to the layered ultrastructure of fenestrated epithelium, GBM, and podocytes, which keeps large bulky particles from entering the tubule (Grahammer 2017; Pavenstadt et al. 2003). Podocytes form elaborate cellular extensions and are connected to adjacent podocytes through cell membrane based protein interactions that create a specialized barrier known as the slit diaphragm (Grahammer 2017). The slit diaphragm does allow small or appropriately charged molecules to pass, which initiates a filtrate product that flows into the tubule and is subsequently augmented by specialized solute transporters arranged in a segmental pattern to create a concentrated waste product (Ichimura et al. 2017; Garg 2018). Within the nephron tubule, the proximal segments perform the bulk of reabsorption, particularly of organic molecules, while the distal segments fine tune the amount of water within the filtrate (Zhuo and Li, 2013). The two kidneys in our body, each composed of around a million nephrons, filter all of the blood in our body almost 30 times daily to produce 1-2 quarts of urine (NIDDK). Any damage or deficit of the specialized cells of the kidney is detrimental to this process, as the human kidney has a limited regenerative capacity. Furthermore, there are currently no therapeutic interventions that can reverse the damage to the kidneys for patients with acquired kidney diseases and birth defects (Wiggins 2007; Romagnani et al. 2017; Reiser and Sever 2013). One reason for this is our limited understanding about kidney developmental pathways.

Early in embryogenesis, the intermediate mesoderm (IM) gives rise to the earliest form of the kidney, known as the pronephros. The paraxial mesoderm (PM) and lateral plate mesoderm (LPM) fields flank the developing IM (Gerlach and Wingert 2013; Perens et al. 2016). While functional in lower vertebrates, the pronephros is vestigial in mammals (Little and McMahon 2012). This structure degenerates to give rise to the mesonephros, which is the terminal kidney in fish and amphibians, but in mammals it is followed by the metanephros (McMahon 2016). Of note, while many organs such as the brain continue to develop post-gestation, humans are born with metanephric kidneys with a static number of nephrons (Luyckx et al. 2011; Little 2016). However, this is not the case for teleosts such as the zebrafish (Danio rerio) that continue to grow nephrons throughout their lifetime and are even capable of neonephrogenesis upon injury (Drummond and Wingert 2016; Drummond and Davidson 2016).

Despite these differences, zebrafish do exhibit fundamental genetic and morphological similarities in kidney organogenesis to mammals. For example, the mammalian renal progenitor markers Lim homeobox 1 (LHX1) and paired box gene 2 (PAX2) are orthologous to LIM homeobox la (lhxla) and paired box $2 a$ (pax2a), which are also renal progenitor markers in zebrafish (Diep et al. 2011, Naylor et al. 2013). Additionally, zebrafish podocytes morphologically resemble mammalian podocytes, and express a suite of markers including Wilms tumor la, Wilms tumor 1b, nephrosis 1, congenital Finnish type (nephrin), nephrosis 2, idiopathic, steroid-resistant (podocin) (wtla/b, $n p h s 1$, and nphs2) that closely correspond to the human homologs WT1, NPHS1, and NPHS2, respectfully (Hsu et al. 2003; Bollig et al. 2006; O’Brien et al., 2011; Zhu et al. 2016). Further, the 
zebrafish nephron exhibits a conserved collection of solute transporter genes that have been shown to be arranged into two proximal and two distal segments similar to other vertebrates including mammals (Figure 1A) (Wingert et al. 2007; Wingert et al. 2011; Desgrange and Cereghini 2015). The genetic conservation combined with the simplicity of the two-nephron and single blood filter pronephros makes the embryonic zebrafish kidney an accessible and powerful genetic model to gain insight into the many puzzles and complexities of kidney development.

A critical regulator of kidney development in both zebrafish and mammals is the zinc-finger transcription factor odd skipped-related 1 (OSR1). In mice, Osrl is one of the earliest markers of the IM and fate-mapping studies have shown that Osrl+ cells differentiate into renal progenitors and renal-associated vasculature (Mugford et al. 2008). Osr1-/- mice fail to express renal progenitors or develop metanephric kidneys, which contributes to embryonic lethality (James et al. 2006; Wang et al. 2005). Similar to mouse studies, zebrafish osrl is an initial marker of the IM (Mugford et al. 2008). Further, knockdown of osrl causes edema, disrupts glomerular morphogenesis, and reduces proximal tubule in both zebrafish and Xenopus (Tena et al. 2007). Subsequent studies have confirmed these findings (Mudumana et al. 2008; Tomar et al. 2014; Neto et al. 2012; Perens et al. 2016; Perens et al. 2020, bioRxiv), though the intriguing observation that osrl knockdown causes the kidney structure to be lost in the region abutting somites 3-5 is not fully understood. In humans, mutations in OSR 1 have been clinically linked to hypomorphic kidneys, making the continued study of this factor and its genetic regulatory network a necessity (Zhang et al. 2011).

In this study, we report the discovery of the zebrafish oceanside (ocn) mutation, which was identified from a forward genetic screen for defects in kidney development based on a striking reduction in podocytes and anterior pronephros tubule (Kroeger et al. 2014; Kroeger et al. 2017; Chambers et al. 2018). Whole genome sequencing revealed the causative lesion of this mutant was a premature stop codon in exon 2 of $o s r 1$. In addition to ocn-/- recapitulating previously observed alterations in mesoderm-derived tissues, reductions in nephron tubule and podocytes were rescued with ectopic osrl cRNA. Interestingly, we found that while osrl was not needed to initially establish the renal progenitor field, osrl was needed to maintain the renal progenitors, as they underwent apoptosis in the absence of Osr1 activity. We also found that wnt2ba transcripts were expressed in podocytes and that this expression was significantly decreased in ocn-/-. Loss of $w n t 2 b a$ led to a reduction in podocytes, and ectopic wnt $2 b a$ was sufficient to partially restore podocyte development in ocn-/-. Further, we placed wnt2ba downstream of the antagonistic influences exerted by osr 1 and hand 2 during IM ontogeny. Together, these data illuminate novel functions of Osr1, which are essential to forwarding our understanding of renal lineage development and may have important implications for congenital kidney defects and diseases as well. 


\section{RESULTS}

ocn encodes a premature stop codon in osr 1 and mutants exhibits defective podocyte and pronephric tubule development

A forward genetic haploid screen was performed to identify regulators of nephrogenesis using the zebrafish pronephros model (Kroeger et al. 2014; Kroeger et al. 2017; Chambers et al. 2018). The ocn mutant was isolated due to its loss of podocytes and anterior pronephros (Figure 1A). Whole mount in situ hybridization (WISH) was performed to delineate the two pronephros tubules based on expression of transcripts encoding cadherin 17 (cdh17) and the podocytes based on wt $1 b$ expression at the 24 and 48 hours post fertilization (hpf) stages (Figure 1A). Both tubule length and podocyte area were found to be significantly reduced in ocn mutants at these time points compared to wild-type (WT) embryos (Figure 1 - figure supplement 1A). By 72 hpf, ocn mutants exhibited dramatic pericardial edema that progressed in severity through $120 \mathrm{hpf}$, and was ultimately lethal (Figure 1B, Figure 1 - figure supplement 1B). Since the kidneys play a major role in fluid homeostasis, this phenotype was a probable indicator of renal dysfunction.

To explore this further, WISH staining to assess tubule and podocyte morphology was conducted on $72 \mathrm{hpf}$ ocn and WT embryos. The animals were embedded in JB-4 plastic resin and serially sectioned. In WT embryos, the blood filter could be detected as a mass of dense capillaries containing glomerular podocytes $(w t 1 b+$ ) flanked by $c d h 17+$ tubules (Figure 1B). In ocn mutants, however, both the glomerulus and the proximal tubules were abrogated (Figure 1B). Instead, a dilated dorsal aorta was identified in this region (Figure 1B).

While it was clear that the proximal pronephros was absent in ocn mutants, it was uncertain if this truncated kidney retained any functionality. Kidney functionality was assessed using an endocytosis assay whereby $70 \mathrm{kDA}$ rhodamine-dextran was injected into the vasculature of ocn::cdh17::GFP embryos, which exhibited a pronephric truncation that phenocopied WISH experiments at 3 days post fertilization (dpf) onwards (Figure 1 - figure supplement 1C). Transgenic animals were injected with rhodamine-dextran at $48 \mathrm{hpf}$ and then assessed at 48 hours post injection (hpi). While dextran was endocytosed in the proximal tubule of non-edemic WT siblings, there was no dextran observed in the truncated tubules of the edemic ocn mutant embryos (Figure 1C). Additionally, we assessed epithelial polarity through immunofluorescence (IF) staining of Na-K-ATPase, which marks transporters localized along the basolateral sides of kidney epithelial cells, and aPKC, which marks the apical side of these epithelia (Gerlach and Wingert 2014). This experiment revealed a similar reduction in tubule and podocytes in ocn as seen with our WISH experiments using the markers $c d h 17 / w t 1 b$ (Figure 1 - figure supplement 1D). Together, this provided strong evidence that the stunted pronephros in ocn-/- was functionally defective.

Next, to identify the causative lesion in ocn, whole genome sequencing was conducted on pools of genomic DNA collected from $24 \mathrm{hpf}$ WISH-identified putative mutants and WT siblings (Leschiner et al. 2012; Ryan et al. 2013). Analysis of the sequencing was performed using SNPtrack software, whereby we detected a strong candidate SNP that was centrally located on chromosome 13 (Figure 1 - figure supplement 2A). Specifically, the putative SNP encoded a missense $\mathrm{C}$ to $\mathrm{T}$ mutation and was predicted to result in an amino acid substitution from an arginine to a premature stop codon in exon 2 of osrl (Figure 1D, Figure 1 - figure supplement 2A). 
To further assess if the predicted stop codon in exon 2 of osr 1 was linked with the ocn phenotype, we performed additional genotyping analysis. For this, genomic DNA was isolated from individual embryos that had been identified as ocn mutants or WTs, based on WISH with the podocyte marker $w t l a$ at $24 \mathrm{hpf}$, and PCR was performed to amplify exon 2 of osr 1 followed by direct Sanger sequencing (Figure 1D). Out of 20 ocn embryos with reduced wtla staining, all 20 were homozygous for the $\mathrm{C}$ to $\mathrm{T}$ mutation in exon 2 of osrl (Figure 1D). Protein alignment showed that zebrafish and human OSR1 protein are 264 amino acids (aa) and 266 aa in length, respectively (Figure 1 - figure supplement 2B). While they exhibit 77\% conservation in overall aa sequence, the three zinc finger domains responsible for DNA binding activity are $100 \%$ conserved across humans, mice and zebrafish (Figure 1 - figure supplement 2B). The osr 1 genetic lesion in ocn-/would place a premature stop codon at residue 165 before all three zinc finger domains (Figure 1 - figure supplement 2B). This suggested that the truncated Osr1 protein produced in ocn-/- would not contain any functional domains and would thus be unable to act as a targeted transcription factor. Next, we verified the effectiveness of a splice-blocking osr 1 morpholino with RT-PCR (Figure 1 - figure supplement 3). osr 1 morphants had a decrease in podocytes and proximal tubule that phenocopied ocn-/- and was consistent with phenotypes reported in previous studies that utilized the same morpholino (Mudumana et al. 2008, Tomar et al. 2014, Neto et al. 2012, Perens et al. 2016) (Figure 1 - figure supplement 3).

Previous literature has indicated that $o s r l$ acts to restrict venous development in order to promote other mesodermal fates such as the kidney and the pectoral fins (Mudumana et al. 2008; Perens et al. 2016; Neto et al. 2012). At 4 dpf, Alcian blue staining indicated that ocn-/- possessed shorter, malformed pectoral fins (Figure 1 - figure supplement 1E). The fin bud area, which gives rise to pectoral fins, was significantly reduced in ocn-/- mutants compared to siblings as seen by the marker MDS1 and EVI1 complex locus (mecom) at $24 \mathrm{hpf}$ (Figure 1 - figure supplement 1F). Additionally, Alcian blue staining revealed altered craniofacial cartilage formation in mutants which fits with previous literature placing osrl as a regulator of palatogenesis in zebrafish and mice (Swartz et al. 2011; Liu et al. 2013) (Figure 1 - figure supplement 1E). In sum, these mesodermal phenotypes were consistent with osrl deficiency.

Next, we evaluated other aspects of pronephros development. As wtla expression appeared to be severely diminished and also disorganized in ocn-/-, we evaluated additional markers to better understand the features of podocyte lineage development in mutants. Podocytes were examined at $24 \mathrm{hpf}$ using a wtla/wtlb double fluorescent in situ (FISH). While clusters of wt $1 a+w t 1 b+$ podocytes were visible in siblings, mutants had a scarcity of double positive cells (Figure 1E). There was also a dearth in $n p h s 1+$ cells, which is a marker of the podocyte slit diaphragm, and suggested that podocyte differentiation was also disrupted (Figure 1F). Additionally, ocn-/embryos displayed diminished pax $2 a$ expression at the 15 ss compared to siblings (Figure $1 \mathrm{G}$ ), again characteristic of osr 1 morphants in previous studies (Mudumana et al. 2008, Neto et al. 2012, Perens et al. 2016).

To test whether the mutation in $o s r l$ was the specific cause of this phenotype, we performed rescue studies. Injection with osrl capped RNA (cRNA) rescued this domain in ocn mutants and expanded it in WT siblings (Figure $1 \mathrm{G}, \mathrm{H}$ ). In sum, ocn mutants reciprocated a multitude of mesodermal phenotypes seen in osrl literature in zebrafish and across taxa. The ability of osrl cRNA to rescue key mesodermal phenotypes in ocn-/- and the catastrophic nature of the osrl mutation, we concluded that Osr1 deficiency is responsible for the ocn phenotype. 


\section{Kidney progenitors are specified in osr1 deficient animals, but subsequently undergo apoptosis}

Previous studies suggest that the anterior pronephros abrogation in osrl zebrafish morphants is due to a fate change where blood/vasculature and endoderm form instead of renal progenitors (Mudumana et al. 2008; Terashima et al. 2014; Perens et al. 2016). Interestingly, in the Osrl mouse knockout model, there was an increase in apoptosis that occurred within the kidney tissue (James et al. 2006). However, in both models, renal progenitors are initially established (James et al. 2006; Mudumana et al. 2008). Thus, we next sought to delineate the cellular dynamics of renal progenitor specification in our ocn mutant model, and to address if alterations in proliferation or apoptosis occur during pronephros development in the absence of osrl.

To investigate this, we first performed WISH studies. The LPM is marked by T-cell acute lymphocytic leukemia 1 (tall), and gives rise to hemangioblasts (Gering et al. 1998; Liao et al. 1998). The IM and hemangioblast domains at the 7 ss were not noticeably different between WT and ocn-/- embryos, as indicated by the markers pax $2 a$, and tall (Figure 2A). However, as previously noted, by the 15 ss there was a decrease in the anterior-most domain of pax $2 a$ expression in ocn-/- embryos (Figure 1G). To further assess the anterior pax $2 a+$ cells between the 7 ss and 15 ss, we performed double FISH studies in WT and ocn-/- embryos to assess pax $2 a$ and tall expression. DAPI staining was also utilized to discern features such as the trunk somites, which allowed for accurate staging. Embryos were flat-mounted and imaged as previously described (Figure 2 - figure 2 supplement 2A) (Cheng et al. 2014). Further, IF was also performed on these samples with anti-caspase-3 antibody to assess the number apoptotic bodies or anti-pH3 to identify proliferating cells. In our analysis we focused on the changes to these markers within somites 1-5, as the IM adjacent to somite 3 gives rise to podocytes (O'Brien et al. 2011).

Beginning at the $7 \mathrm{ss}$, ocn-/- embryos exhibited a significant increase in the number of caspase-3+ fragments within the combined tall and pax $2 a$ fields near somites 1-5 (Figure 2B). However, by the $15 \mathrm{ss}$, few apoptotic fragments were visible in the area of interest, with no significant differences between WT and ocn-/- embryos (Figure 2B). Similar to the $7 \mathrm{ss}$, we found a significant increase in the number of total caspase-3+ fragments at the $10 \mathrm{ss}$ in ocn-/- mutants and osr 1 morphants, while the WT siblings had little to no apoptosis occurring in this area (Figure 2C-E). Interestingly, most of the apoptosis that occurred in mutants and morphants happened within the pax $2 a$ kidney field specifically (Figure 2E). Another finding of note was that the number of caspase-3+ fragments was not significantly different between osr 1 morphants and ocn-/- for either assessment (Figure 2D,E).

To further understand the cell dynamics across this time course, absolute area measurements of pax $2 a$ and tall were taken at 7, 10, and 15 ss from somites 1-5 in WT and ocn-/-. Surprisingly, the area of the tall domain was already expanded at $7 \mathrm{ss}$, and continued to expand through the $15 \mathrm{ss}$ (Figure 2 - figure supplement 1). However, across the three time points examined, a reduction in pax $2 a$ area was only significantly different between WT and ocn-/- at the 15 ss (Figure 2 - figure supplement 1). Further, although the tall field was expanded in ocn-/- embryos at the $10 \mathrm{ss,} \mathrm{there}$ was no significant difference in proliferating $\mathrm{pH} 3+$ cells between WT and osrl loss of function models (Figure 2F,G). Additionally, no significant changes in proliferation were seen between mutants and WTs at the 8 ss (Figure 2 - figure supplement 1). 
To determine if apoptosis was occurring within podocyte progenitors in the pax $2 a$ kidney field, we performed an additional FISH with wt $1 a$ and pax2a at 10 ss. During this time point, while pax $2 a$ expression begins adjacent to somite $3, w t l a$ is expressed from somites 1-3 (Figure $2 \mathrm{H}$ ). Similar to the pax $2 a$ domain, the wtla domain did not appear to be reduced at this time point, though it does become restricted and disorganized by $24 \mathrm{hpf}$ (Figure 1D). We found a significant increase in caspase-3+ fragments that were double positive for wt la and pax2a in osr 1 morphants compared to WTs (Figure 2H,I). These results demonstrated that abnormal apoptosis occurred in podocyte progenitors due to loss of osrl. In sum, osr 1 is not needed to initiate the pax $2 a$ progenitor pool, but it is needed to maintain this population, including the podocyte precursors, during pronephros development.

\section{Ectopic osr 1 is transiently sufficient to rescue renal progenitors}

Our observation that pax $2 a+$ renal progenitors arise in ocn mutants, but are not maintained, is consistent with previous data that osr 1 knockdown leads to a reduced pax $2 a+$ renal progenitor field by the 14 ss (Mudumana et al. 2008). As pax2a expression marks both podocyte and tubule precursors (O'Brien et al. 2011), we hypothesized that osrl is likely needed for podocyte and tubule progenitor maintenance. To determine this, we performed a series of rescue studies in our ocn mutants to test if one or both of these compartments requires osrl for its maintenance.

First, we tested whether overexpression of osrl mRNA was sufficient to rescue podocytes in ocn mutants by assessing $w t 1 b$ expression, which specifically marks the podocyte lineage (Bollig et al. 2006; O'Brien et al. 2011). Provision of $o s r 1$ mRNA robustly rescued the development of $w t 1 b+$ podocytes in ocn mutants at the 15 ss (Figure 3A,B). However, by the 22 ss, we were only able to achieve a partial podocyte rescue, though tubules within the same individuals appeared to be WT in length (Figure 3C,D). Consistent with this, we were unable to obtain a podocyte rescue at 24 hpf (data not shown), though again we could achieve a rescue of the truncated tubules (Figure $3 \mathrm{E}, \mathrm{F})$. Interestingly, overexpression of $o s r l$ was sufficient to induce ectopic $c d h 17+$ cells in about $55 \%$ of injected embryos (Figure $3 \mathrm{G}$ ). It should be noted that osr 1 cRNA did lead to a decrease in body axis length when compared to uninjected WTs and mutants, which in turn affected pronephros length measurements (Figure 3 - figure supplement 1A). Despite this, the percentage of kidney length to body length was not significantly different between embryos injected with osrl cRNA and uninjected animals.

We also conducted a rescue time course by co-injecting osr $1 \mathrm{MO}$ and osr 1 cRNA and performed WISH using $c d h 17$ to assess the tubule during a number of stages. While $90 \%$ of animals injected with both constructs exhibited a WT tubule length at $24-27$ ss, by $36 \mathrm{hpf}$, only $50 \%$ showed a rescue (Figure 3H, Figure 3 - figure supplement 1B). At 48 hpf, only $20 \%$ of injected embryos had a WT length pronephric tubules while $80 \%$ had a unilateral or bilateral reduction (Figure $3 \mathrm{H}$, Figure 3 - figure supplement 1B). Together, this indicated that the pronephros requires a continued presence of $\mathrm{osrl}$ in order for the tubule population to be maintained as development progressed. 


\section{wnt2ba is a novel podocyte marker and regulator}

Given the importance of $o s r l$ to podocyte development and maintenance, we wanted to identify downstream factors that promote podocytes. It was previously shown that the canonical Wnt ligand wingless-type MMTV integration site family, member $2 \mathrm{Ba}$ (wnt2ba) is expressed in a similar proximal swath of IM as osrl (Neto et al. 2012). We observed a similar expression pattern of $w n t 2 b a$ in the anterior IM as early as 13 ss (Figure 4A). To specifically determine which cells $w n t 2 b a$ was expressed in, we conducted FISH studies. At the 20-22 ss, wnt2ba transcripts were colocalized in cells within the anterior most region of pax $2 a+$ and $w t 1 b+$ IM (Figure 4B, Figure 4 - figure supplement $1 \mathrm{~A})$. At $15 \mathrm{ss}$, wnt $2 b a$ transcripts were also colocalized with $w t 1 a / b+$ podocyte progenitor cells (Figure 4 - figure supplement 1A,B). At $24 \mathrm{hpf}$, wnt $2 b a$ was expressed in both $w t 1 b+$ podocyte precursor cells and in neighboring cells of the IM (Figure 4C). By $48 \mathrm{hpf}, w n t 2 b a$ was restricted to the podocytes and overlapped precisely with $w t 1 b$ expression (Figure 4C). Taken together, we conclude that wnt2ba is a novel podocyte marker. We also examined the expression of the zebrafish wnt2ba paralogue, wnt2bb, at 24 hpf using FISH, but determined that transcripts were located anterior to the podocyte and kidney fields (Figure 4 - figure supplement 2).

Given its expression in podocyte progenitors, we hypothesized that Wnt2ba might have roles in podocyte specification or differentiation. To explore whether wnt $2 b a$ is needed for proper podocyte formation, we performed wnt $2 b a$ loss of function studies. We first verified a morpholino that blocked splicing at exon 1, as well as a morpholino that targeted the start site (Figure 4 - figure supplement 3). When wnt2ba morphants were examined at $24 \mathrm{hpf}$, there was a significant reduction in the expression of $w t 1 b$ and $n p h s 1$ that corresponded to a smaller podocyte area and net cell number. (Figure 4D-H). We also found that the area of $w t 1 a+/ w t 1 b+$ coexpressing podocytes was decreased in wnt $2 b a$ morphants at $24 \mathrm{hpf}$ (Figure 4I,J). Furthermore, the decrease in podocyte number in wnt $2 b a$ morphants occurred between the 15 and 22 ss, suggesting wnt $2 b a$ is required to maintain the podocyte lineage (Figure 4 - figure supplement 4). In contrast, wnt $2 b a$ morphants showed no discernable changes in development or maintenance of the $c d h 17+$ nephron tubule (Figure 4 - figure supplement $3 \mathrm{E}$ ). Collectively, these data lead us to conclude that wnt $2 b a$ is a significant regulator of podocyte ontogeny.

\section{osr 1 promotes wnt $2 b a$ in the podocyte developmental pathway}

Previous research has demonstrated that osrl morphants exhibited a dramatic decrease in the $w n t 2 b a$ pronephric domain, though wnt2ba morphants had no notable change in osr 1 expression (Neto et al. 2012). They postulated that osrl acts to promote wnt2ba in the IM which allows for proper pectoral fin development to occur (Neto et al. 2012). This led us to hypothesize that this same genetic cascade in the IM promotes the formation of proximal pronephric tissues, such as the podocytes, and is dysfunctional in ocn-/-.

In congruence with this prior study, we found that wnt $2 b a$ is significantly reduced in ocn-/- at both $15 \mathrm{ss}$, and is almost completely absent by $24 \mathrm{hpf}$ (Figure 5A). We also observed that wnt $2 b a$ and osr 1 transcripts were colocalized in a population of presumptive IM cells at 15 ss and 22 ss, putting them in the right place and the right time to interact (Figure 5B, Figure 5 - figure supplement 1). Overexpression of wnt $2 b a$ led to an increase in podocyte number and domain area in injected WT 
embryos, as seen with an increase in the markers $w t 1 b$ and $n p h s 1$ (Figure 5C-H). While injection with osr $1 \mathrm{MO}$ alone leads to diminished podocytes, co-injection of wnt $2 b a$ cRNA with osrl MO led to a rescue in podocyte area and cell count (Figure 5C-H). Together, this indicates that wnt $2 b a$ is sufficient to drive podocyte development, and does so downstream of osrl.

\section{hand2 suppresses podocyte development by restricting wnt $2 b a$ expression and podocyte development}

The bHLH transcription factor heart and neural crest derivatives expressed 2 (hand2) has been shown to be antagonistic to osrl in early mesoderm development (Perens et al. 2016). The loss of osrl leads to decreases in podocytes and tubules and an increase in hemangioblasts; in contrast, loss of hand 2 results in expansions in renal cells at the expense of vasculature (Perens et al. 2016). Concomitant knockdown of osrl and hand2 rescues tubule development (Perens et al. 2016) and podocyte development (Perens et al. 2020, bioRxiv).

When we knocked down hand 2 using an ATG morpholino, we observed a separation in the myosin light chain 7 ( $m y l 7$ ) heart field at 22 ss that matched previously observed phenotypes (Maves et al. (2009) (Figure 6 - figure supplement 1). Knockdown of hand2 also caused a significant increase in $w t 1 b+$ podocyte domain area and cell number (Figure 6A-C). While uninjected ocn-/- embryos had little to no podocytes, injecting ocn-/- with hand2 MO resulted in an expansion in podocyte number and area that was significantly different from mutants (Figure 6A-C). Similarly, hand2 morphants had a significantly larger wnt2ba domain, and hand2/osrl MO coinjection rescued the usually abrogated wnt $2 b a$ domain (Figure 6D,E). This indicated that imbalance of hand 2 and $o s r l$ leads to changes in wnt2ba expression, which alters podocyte development. 


\section{DISCUSSION}

While there are dozens to hundreds of podocyte diseases and maladies that have been characterized, the genetic explanations for their origin and progression is lacking. One reason for this is that there are relatively few factors that are known to promote the development of these specialized epithelia. Continuing to identify these factors is critical for future diagnostics and treatments for podocytopathy. In this study, we have both re-examined a previous factor shown to promote podocyte fates, osrl, and identified a new downstream regulator, wnt $2 b a$.

ocn was identified as a mutant of interest in a forward genetic screen due to displaying pericardial edema and decreases in podocytes and proximal tubule. Through a whole genome sequencing approach, we determined that ocn-/- harbors a SNP in exon 2 that leads to a premature stop in $o s r 1$. This SNP was confirmed as the causative lesion in ocn-/- when osrl cRNA could rescue each of these phenotypes. Upon confirmation that ocn was an osrl mutant line, we next sought to fully assess how osrl loss of function impacts kidney development in the context of a zebrafish mutant. We found that osrl is needed to maintain renal progenitors and inhibit the development of hemangioblasts.

Further, we established a genetic pathway controlled by osrl that regulate podocyte survival by promoting wnt2ba expression. We found that wnt $2 b a$ is an IM/podocyte marker that is likewise diminished in ocn-/-. Loss and gain of wnt $2 b a$ leads to a decrease and increase in podocyte area, demonstrating that wnt $2 b a$ is both necessary and sufficient to drive podocyte development. Notably, wnt $2 b a$ can rescue podocytes in an osrl-deficient background, which places this factor downstream of osrl (Figure 6F). Finally, the osr1/wnt2ba podocyte pathway is negatively regulated by hand2 (Figure $6 \mathrm{~F})$.

\section{osr1 acts to promote podocytes}

The earliest known podocyte marker in zebrafish is $w t 1 a$, though the paralogue, wt $1 b$, that appears at $12 \mathrm{ss}$ is expressed in a more specific territory (Bollig et al. 2006; Bollig et al. 2009). It has also been suggested that wtla is more dominant than wtlb, as knockdown of wtla leads to loss of $n p h s 1 / 2$ while knockdown of $w t 1 b$ causes less dramatic podocyte phenotypes (Perner et al. 2007). Zebrafish literature has shown that $o s r 1$ morphants exhibit reductions in $w t 1 b, l h x l a$, and $n p h s 1 / 2$ at $24 \mathrm{hpf}$ that have also been observed in ocn-/- (Mudumana et al. 2008; Tomar et al. 2014, Tena et al. 2007). However, the relationship between wtla and osrl has yet to be fully understood. Tomar et al. (2014) placed wtla upstream of osrl due to osrl being reduced in wt1 morpholinoinjected embryos and wtla expression being interpreted as "unchanged" in osr 1 morpholinoinjected animals. However, in our studies, osrl morphants do exhibit alterations in wtla+ cell organization and a restriction in domain that phenocopies ocn-/-. Mouse studies have shown that $W T 1+/-; O S R 1+/-$ mice exhibit reduced kidneys, suggesting that these factors act cooperatively in kidney and podocyte development (Xu et al. 2016). If osr 1 and $w t 1 a$ did have a similar synergistic relationship in zebrafish kidney development, this would also explain reports that wt 1 morphants exhibit a loss of podocytes and proximal tubules reminiscent of osr 1 loss of function models (Tomar et al. 2014). While there are currently limitations in using anti-Osr1 antibodies in any in vivo model, progress in this area is needed in order to ascertain if wtla and osrl are directly interacting during kidney development. 


\section{osr1 is needed for kidney cell maintenance}

While ocn-/- exhibit normal patterning of IM early in development, by the time specification to pronephros is beginning to occur around $15 \mathrm{ss}$, the anterior domain is absent. Our experiments demonstrated that this is due to two events; (1) an expansion of hemangioblasts, and (2) apoptosis of podocyte progenitors in this region. Work in chick and mouse has shown that while mesonephric tissues and markers are present, apoptosis occurs within nephrogenic mesenchyme that keeps metanephric tissues from forming in Osrl knockout animals (Wang et al. 2005; James et al. 2006). Further, previous studies have shown that $O s r l$ acts synergistically with factors such as $W t 1$ and Six2 to renew renal stem cell pools to inhibit premature differentiation and thus cell death (Xu et al. 2014). A similar apoptosis event has not been recorded in osrl loss of function zebrafish models prior to this study, and we hypothesize that osrl plays a similar role in progenitor self-renewal in zebrafish.

The expansion in hemangioblast domain in osrl morphants has been documented by other groups, where it was suggested that pax $2 a+$ cells were converting to tal+ cells (Mudumana et al. 2008). Additionally, the expansion in vessel progenitors has been reported in an osr 1 TALEN mutant (Perens et al. 2020, bioRxiv). However, our results add one further element to these early events, as we have captured cell apoptosis in pax $2 a+$ cells of osr 1 mutant embryos. Further, our studies have revealed that the timing of the pax $2 a$ domain decrease and hemangioblast domain increase is not equivalent. The hemagioblasts expand hours prior to the loss of the anterior IM domain. We postulate that osrl may inhibit hemangioblast formation either indirectly or in an independent mechanism than it uses to promote IM and podocytes.

\section{wnt2ba is a novel regulator of podocyte development}

$w n t 2 b a$ is a ligand that functions in the canonical Wnt/beta-catenin pathway. As a member of this pathway, wnt $2 b a$ acts to promote cell growth, differentiation and migration during development. In regards to kidney development, it has been shown that $W n t 2 b$ can be detected in the kidney stroma in mice as early as E11.5 (Lin et al. 2001; Iglesias et al. 2007), and in humans WNT2B is expressed in fetal kidney stroma (Combes et al., 2019). In addition, cells expressing Wnt $2 b$ promote ureteric branching in culture (Lin et al. 2001). Wnt2/2b is also paramount to normal lung and pectoral fin development in both aquatic and mammalian species (Goss et al. 2009, Neto et al. 2012). Interestingly, osrl has been shown to act downstream of retinoic acid signaling yet upstream of wnt $2 b$ in both pectoral fin development in zebrafish (Neto et al. 2012) and in lung progenitor specification in foregut endoderm in Xenopus (Rankin et al. 2012). However, our study has both evaluated the role of wnt $2 b a$ as a regulator of kidney development and placed its function downstream of osrl to specifically promote the podocyte lineage. Further, we show that $o s r l$ promotes wnt $2 b a$ expression during podocyte development through a mechanism involving the inhibition of hand2. In synchrony with our data, a recent report similarly found that reciprocal antagonism between osrl and hand2 is essential for the normal emergence of $w t 1 b+$ podocyte precursors (Perens et al. 2020, bioRxiv). 
We show in the present study that wnt2ba is a regulator of podocyte development, but that loss of wnt2ba does not cause compelling changes in PCT or tubule length. Another study by Lyons et al. (2009) showed that broad inhibition of Wnt signaling through heat-shock activation of $d k k 1$ lead to an abrogation in the zebrafish pronephros that resembles osrl loss of function models. Wnt ligands are highly regionalized to allow for precise regulation during tissue development (Iglesias 2007; Verkade and Heath 2008). Our findings that wnt $2 b a$ is restricted to the podocytes by $48 \mathrm{hpf}$ could reflect regional specificity. This suggests that there are other Wnt ligands and receptors that act to regulate certain kidney lineages in zebrafish development. Loss of one or more of these factors in combination with wnt2ba could lead to an anterior truncation of the pronephros that resembles the experiments from Lyons et al. (2009). Future studies are needed to discern these factors and additional downstream targets of both wnt $2 b a$ and $o s r l$.

Taken together, these results have allowed us to garner new insights into podocyte development in zebrafish. By selecting ocn as a mutant of interest from our ENU screen, we have discovered an osr 1 mutant and confirmed its significance in zebrafish pronephros development in an unbiased manner. We have expanded on these previous findings by demonstrating that osrl is required for to inhibit apoptosis in specified kidney precursors, and later for nephron cell maintenance. We have also ascertained new roles for osrl in promoting wnt $2 b a$ expression, which it does in part through antagonism of hand2. Finally, our results show that wnt $2 b a$ mitigates podocyte development downstream of the osr1/hand 2 interaction. Given how little is known about CAKUT and kidney agenesis, findings from genetics studies such as the present work are crucial to furthering our understanding about the causes and solutions to these disease states. 


\section{METHODS}

\section{Creation and maintenance of zebrafish lines}

Zebrafish were housed in the Center for Zebrafish Research in the Freimann Life Science Center at the University of Notre Dame. All experiments and protocols used in this study were approved by the Institutional Animal Care and Use Committee (IACUC). We performed an ENU haploid genetic screen as described in Kroeger et al. (2017) and Chambers et al. (2018).

\section{Live imaging and dextran injections}

Embryos were grown in E3 media at approximately $28^{\circ} \mathrm{C}$. For live imaging, embryos were placed in a solution of $2 \%$ methylcellulose/E3 and $0.02 \%$ tricaine and placed in a glass depression slide. For dextran injection experiments, embryos were also incubated with $0.0003 \%$ phenylthiourea (PTU) in E3 to inhibit pigment development. At 3 dpf, ocn::cdh17::GFP animals were anesthetized and injected with $40 \mathrm{kDA}$ rhodamine-dextran. Embryos were then examined and imaged 24 hours after injection.

\section{WISH, FISH, IF, sectioning and image acquisition}

WISH was performed as described in previous studies (Wingert et al. 2007, Wingert and Davidson 2011, Li et al. 2014, Cheng and Wingert 2015). For each marker, embryos from at least 3 sets of parents were assessed and 5 mutants and 5 siblings were imaged. Immunocytochemistry (ICC) was performed as previously described (Kroeger et al. 2017, Marra et al. 2018). Embryos from WISH experiments were embedded in JB-4 plastic blocks and cut to obtain $4 \mu \mathrm{m}$ sections which were counterstained with methylene blue (0.5\%) to mark nuclei. Alcian blue staining was performed as described (Chambers et al. 2018).

\section{Genotyping}

Direct genotyping on ocn fin clips and embryos was carried out by PCR amplification of exon 2 of osrl (Forward primer: CCCCATTCACTTTGCCACGCTGCACCTTTTC, reverse primer: CTGTGGTCTCTCAGGTGGTCCTGCCTCCTAAA). Dilutions of purified PCR products were then subjected to Sanger sequencing by the Genomics Core at University of Notre Dame using the forward primer.

\section{Morpholinos and RT-PCR}

osr1 morpholino (ATCTCATCCTTACCTGTGGTCTCTC) was first described in Mudumana et al. (2008) and was designed to block the splice donor site of exon 2. We used the primers GTGACTGTATCTGAATCCTCTTATTTTGGATCGTCTCGCTTCACAAAGAACTG, and CTGTAGGCTATGGAAGTTTGCCTTTTCAGGAAGCTCTTTGGTCAG to perform RT-PCR as described in (Kroeger et al. 2017) to confirm the interruption of exon 2 splicing activity. wnt $2 b a$ splice blocking morpholino (CTGCAGAAACAAACAGACAATTAAG) was previously utilized 
in Neto et al. (2012). However, we did not find their methods of RT-PCR to be specific to wnt2ba transcripts, so we designed RT-PCR primers to amplify the entire transcript; (Forward primer: ATGCCAGAGTGTGATGGAGTTGGGTGCGCGTCGCCGGCGC, (Reverse primer: GCTGGAGCGAGACCACACTGTGTTCGGCCGC), and additionally looked for the presence of intronic sequence with (Intronic forward primer: ATCACAGGGGTATCATTATCACAAAAAATTGTAAATAAATG). While this splice blocking morpholino was the primary method of wnt $2 b a$ knockdown, a wnt $2 b a$ ATG morpholino (ACCCAACTCCATCACACTCTGGCAT, Wakahara et al. 2007) was used to confirm phenotypes seen with the splicing morpholino. The hand2 ATG morpholino (CCTCCAACTAAACTCATGGCGACAG) was used as described in Maves et al. (2009).

\section{Statistics and measurements}

Absolute domain lengths and area measurements were taken from five representative embryos per treatment using Fiji is just ImageJ. Averages, standard deviation, and unpaired student's t-tests were performed in Microsoft Xcel and GraphPad Prism. In experiments where osrl cRNA was used, body axis measurements were taken for injected and uninjected embryos. The tubule measurements for each group was divided by the body length measurement to discern what percentage of the body length was occupied by the kidney. To normalize the data, these percentages were subjected to arcsine degree transformation and then run through a student's t-test to determine significance. 


\section{References}

Bollig, F., Mehringer, R., Perner, B., Hartung, C., Schäfer, M., Schartl, M., Volff, J., Winkler, C., Englert, C., 2006. Identification and Comparative Expression Analysis of a Second Wt1 Gene in Zebrafish. Developmental dynamics : an official publication of the American Association of Anatomists 235, 554.

Chambers, J.M., Poureetezadi, S.J., Addiego, A., Lahne, M., Wingert, R.A., 2018. Ppargc1a Controls Nephron Segmentation during Zebrafish Embryonic Kidney Ontogeny. Elife.

Cheng, C.N., Wingert, R.A., 2015. Nephron Proximal Tubule Patterning and Corpuscles of Stannius Formation are Regulated by the Sim1a Transcription Factor and Retinoic Acid in Zebrafish. Dev Biol 399, 100-116.

Cheng, C.N., Li, Y., Marra, A.N., Verdun, V., Wingert, R.A., 2014. Flat Mount Preparation for Observation and Analysis of Zebrafish Embryo Specimens Stained by Whole Mount in Situ Hybridization. J Vis Exp .

Combes, A.N., Combes, A.N., Zappia, L., Er, P.X., Oshlack, A., Little, M.H. 2019. Single-cell Analysis Reveals Congruence Between Kidney Organoids and Human Fetal Kidney. Genome Med 11, 3.

Desgrange, A., Cereghini, S., 2015. Nephron Patterning: Lessons from Xenopus, Zebrafish, and Mouse Studies. Cells 4, 483-499.

Diep, C.Q., Ma, D., Deo, R.C., Holm, T.M., Naylor, R.W., Arora, N., Wingert, R.A., Bollig, F., Djordjevic, G., Lichman, B., Zhu, H., Ikenaga, T., Ono, F., Englert, C., Cowan, C.A., Hukriede, N.A., Handin, R.I., Davidson, A.J., 2011. Identification of Adult Nephron Progenitors Capable of Kidney Regeneration in Zebrafish. Nature 470, 95-100.

Drummond, B.E., Wingert, R.A., 2016. Insights into Kidney Stem Cell Development and Regeneration using Zebrafish. World J Stem Cells 8, 22-31.

Drummond, I.A., Davidson, A.J., 2016. Zebrafish Kidney Development. Methods in Cell Biology 134, 391-429.

Garg, P., 2018. A Review of Podocyte Biology. Am J Nephrol 47 Suppl 1, 3-13.

Gering, M., Rodaway, A.R., Göttgens, B., Patient, R.K., Green, A.R., 1998. The SCL Gene Specifies Haemangioblast Development from Early Mesoderm. EMBO J 17, 4029-4045.

Gerlach, G.F., Wingert, R.A., 2013. Kidney Organogenesis in the Zebrafish: Insights into Vertebrate Nephrogenesis and Regeneration. Wiley Interdisciplinary Reviews: Developmental Biology 2, 559-585.

Gerlach, G.F., Wingert, R.A., 2014. Zebrafish Pronephros Tubulogenesis and Epithelial Identity Maintenance are Reliant on the Polarity Proteins Prkc Iota and Zeta. Dev Biol 396, 183-200.

Goss, A.M., Tian, Y., Tsukiyama, T., Cohen, E.D., Zhou, D., Lu, M.M., Yamaguchi, T.P., Morrisey, E.E., 2009b. Wnt2/2b and B-Catenin Signaling are Necessary and Sufficient to Specify Lung Progenitors in the Foregut. Developmental Cell 17, 290-298. 
Grahammer, F., 2017. New Structural Insights into Podocyte Biology. Cell Tissue Res 369, 5-10.

Hsu, H., Lin, G., Chung, B., 2003. Parallel Early Development of Zebrafish Interrenal Glands and Pronephros: Differential Control by Wt1 and Ff1b. Development 130, 2107-2116.

Ichimura, K., Kakuta, S., Kawasaki, Y., Miyaki, T., Nonami, T., Miyazaki, N., Nakao, T., Enomoto, S., Arai, S., Koike, M., Murata, K., Sakai, T., 2017. Morphological Process of Podocyte Development Revealed by Block-Face Scanning Electron Microscopy. J Cell Sci 130, 132-142.

Ichimura, K., Miyazaki, N., Sadayama, S., Murata, K., Koike, M., Nakamura, K., Ohta, K., Sakai, T., 2015. Three-Dimensional Architecture of Podocytes Revealed by Block-Face Scanning Electron Microscopy. Sci Rep 5, 8993.

Iglesias, D.M., Hueber, P., Chu, L., Campbell, R., Patenaude, A., Dziarmaga, A.J., Quinlan, J., Mohamed, O., Dufort, D., Goodyer, P.R., 2007. Canonical WNT Signaling during Kidney Development. Am J Physiol Renal Physiol 293, 494.

James, R.G., Kamei, C.N., Wang, Q., Jiang, R., Schultheiss, T.M., 2006. Odd-Skipped Related 1 is Required for Development of the Metanephric Kidney and Regulates Formation and Differentiation of Kidney Precursor Cells. Development 133, 2995-3004.

Kroeger, P.T., Drummond, B.E., Miceli, R., McKernan, M., Gerlach, G.F., Marra, A.N., Fox, A., McCampbell, K.K., Leshchiner, I., Rodriguez-Mari, A., BreMiller, R., Thummel, R., Davidson, A.J., Postlethwait, J., Goessling, W., Wingert, R.A., 2017. The Zebrafish Kidney Mutant Zeppelin Reveals that Brca2/Fancd1 is Essential for Pronephros Development. Developmental Biology 428, 148-163.

Kroeger, P.T., Poureetezadi, S.J., McKee, R., Jou, J., Miceli, R., Wingert, R.A., 2014. Production of Haploid Zebrafish Embryos by in Vitro Fertilization. J Vis Exp.

Kroeger, P.T., Wingert, R.A., 2014. Using Zebrafish to Study Podocyte Genesis during Kidney Development and Regeneration. genesis 52, 771-792.

Leshchiner, I., Alexa, K., Kelsey, P., Adzhubei, I., Austin-Tse, C.A., Cooney, J.D., Anderson, H., King, M.J., Stottmann, R.W., Garnaas, M.K., Ha, S., Drummond, I.A., Paw, B.H., North, T.E., Beier, D.R., Goessling, W., Sunyaev, S.R., 2012. Mutation Mapping and Identification by Whole-Genome Sequencing. Genome Res 22, 1541-1548.

Li, Y., Cheng, C.N., Verdun, V.A., Wingert, R.A., 2014. Zebrafish Nephrogenesis is Regulated by Interactions between Retinoic Acid, Mecom, and Notch Signaling. Developmental biology $386,111-122$.

Liao, E.C., Paw, B.H., Oates, A.C., Pratt, S.J., Postlethwait, J.H., Zon, L.I., 1998. SCL/Tal-1 Transcription Factor Acts Downstream of Cloche to Specify Hematopoietic and Vascular Progenitors in Zebrafish. Genes Dev 12, 621-626.

Lin, Y., Liu, A., Zhang, S., Ruusunen, T., Kreidberg, J.A., Peltoketo, H., Drummond, I., Vainio, S., 2001a. Induction of Ureter Branching as a Response to Wnt-2b Signaling during Early Kidney Organogenesis. Developmental dynamics : an official publication of the American Association of Anatomists 222, 26-39. 
Little, M.H., 2016. Growing Kidney Tissue from Stem Cells: How Far from "Party Trick" to Medical Application? Cell Stem Cell 18, 695-698.

Little, M.H., McMahon, A.P., 2012. Mammalian Kidney Development: Principles, Progress, and Projections. Cold Spring Harb Perspect Biol 4, .

Liu, H., Lan, Y., Xu, J., Chang, C., Brugmann, S.A., Jiang, R., 2013. Odd-Skipped Related-1 Controls Neural Crest Chondrogenesis during Tongue Development. Proc Natl Acad Sci U S A $110,18555-18560$.

Luyckx, V.A., Shukha, K., Brenner, B.M., 2011. Low Nephron Number and its Clinical Consequences. Rambam Maimonides medical journal 2, e0061.

Lyons, J.P., Miller, R.K., Zhou, X., Weidinger, G., Deroo, T., Denayer, T., Park, J., Ji, H., Hong, J.Y., Li, A., Moon, R.T., Jones, E.A., Vleminckx, K., Vize, P.D., McCrea, P.D., 2009.

Requirement of Wnt/B-Catenin Signaling in Pronephric Kidney Development. Mechanisms of Development 126, 142-159.

Marra, A.N., Ulrich, M., White, A., Springer, M., Wingert, R.A., 2017. Visualizing Multiciliated Cells in the Zebrafish through a Combined Protocol of Whole Mount Fluorescent in Situ Hybridization and Immunofluorescence. J Vis Exp .

Maves, L., Tyler, A., Moens, C.B., Tapscott, S.J., 2009. Pbx Acts with Hand2 in Early Myocardial Differentiation. Dev Biol 333, 409-418.

McMahon, A.P., 2016. Development of the Mammalian Kidney. Current topics in developmental biology 117, 31 .

Mugford, J.W., Sipilä, P., McMahon, J.A., McMahon, A.P., 2008. Osr1 Expression Demarcates a Multi-Potent Population of Intermediate Mesoderm that Undergoes Progressive Restriction to an Osr1-Dependent Nephron Progenitor Compartment within the Mammalian Kidney. Dev Biol 324, 88-98.

Naylor, R.W., Przepiorski, A., Ren, Q., et al., 2013. HNF1B is essential for nephron segmentation during nephrogenesis. J. Am. Soc. Nephrol. 24, 77-87.

Naylor, R.W., Dodd, R.C., Davidson, A.J., 2016. Caudal Migration and Proliferation of Renal Progenitors Regulates Early Nephron Segment Size in Zebrafish. Scientific Reports (Nature Publisher Group) 6, 35647.

Neto, A., Mercader, N., Gómez-Skarmeta, J.L., 2012. The Osr1 and Osr2 Genes Act in the Pronephric Anlage Downstream of Retinoic Acid Signaling and Upstream of Wnt2b to Maintain Pectoral Fin Development. Development (Cambridge, England) 139, 301-311.

O'Brien, L.L., Grimaldi, M., Kostun, Z., Wingert, R.A., Selleck, R., Davidson, A.J., 2011. Wt1a, Foxc1a, and the Notch Mediator Rbpj Physically Interact and Regulate the Formation of Podocytes in Zebrafish. Developmental Biology 358, 318-330.

Perens, E.A., Garavito-Aguilar, Z.V., Guio-Vega, G.P., Peña, K.T., Schindler, Y.L., Yelon, D., 2016. Hand2 Inhibits Kidney Specification while Promoting Vein Formation within the Posterior Mesoderm. eLife 5. 
Perens E.A., Diaz J.T., Quesnel A., Crump, J.G., Yelon, D. 2020. osr1 couples intermediate mesoderm cell fate with temporal dynamics of vessel progenitor cell differentiation. bioRxiv https://doi.org/10.1101/2020.11.14.383141

Perner, B., Englert, C., Bollig, F., 2007. The Wilms Tumor Genes Wt1a and Wt1b Control Different Steps during Formation of the Zebrafish Pronephros. Dev Biol 309, 87-96.

Rankin, S.A., Gallas, A.L., Neto, A., Gómez-Skarmeta, J.L., Zorn, A.M., 2012. Suppression of Bmp4 Signaling by the Zinc-Finger Repressors Osr1 and Osr2 is Required for Wnt/B-CateninMediated Lung Specification in Xenopus. Development 139, 3010-3020.

Reiser, J., Sever, S., 2013. Podocyte Biology and Pathogenesis of Kidney Disease. Annual Review of Medicine 64, 357-366.

Romagnani, P., Remuzzi, G., Glassock, R., Levin, A., Jager, K.J., Tonelli, M., Massy, Z., Wanner, C., Anders, H., 2017. Chronic Kidney Disease. Nature Reviews Disease Primers 3, 17088 .

Ryan, S., Willer, J., Marjoram, L., Bagwell, J., Mankiewicz, J., Leshchiner, I., Goessling, W., Bagnat, M., Katsanis, N., 2013. Rapid Identification of Kidney Cyst Mutations by Whole Exome Sequencing in Zebrafish. Development 140, 4445-4451.

Swartz, M.E., Sheehan-Rooney, K., Dixon, M.J., Eberhart, J.K., 2011. Examination of a Palatogenic Gene Program in Zebrafish. Developmental dynamics : an official publication of the American Association of Anatomists 240, 2204-2220.

Tena, J.J., Neto, A., de la Calle-Mustienes, E., Bras-Pereira, C., Casares, F., Gómez-Skarmeta, J.L., 2007. Odd-Skipped Genes Encode Repressors that Control Kidney Development. Developmental Biology 301, 518-531.

Terashima, A.V., Mudumana, S.P., Drummond, I.A., 2014. Odd Skipped Related 1 is a Negative Feedback Regulator of Nodal-induced Endoderm Development. Developmental Dynamics 243, 1571-1580.

Tomar, R., Mudumana, S.P., Pathak, N., Hukriede, N.A., Drummond, I.A., 2014. Osr1 is Required for Podocyte Development Downstream of Wt1a. Journal of the American Society of Nephrology : JASN 25, 2539-2545.

Verkade, H., Heath, J.K., 2008. Wnt Signaling Mediates Diverse Developmental Processes in Zebrafish. Methods in molecular biology (Clifton, N.J.) 469, 225.

Wakahara, T., Kusu, N., Yamauchi, H., Kimura, I., Konishi, M., Miyake, A., Itoh, N., 2007. Fibin, a Novel Secreted Lateral Plate Mesoderm Signal, is Essential for Pectoral Fin Bud Initiation in Zebrafish. Dev Biol 303, 527-535.

Wang, Q., Lan, Y., Cho, E., Maltby, K.M., Jiang, R., 2005. Odd-Skipped Related 1 (Odd 1) is an Essential Regulator of Heart and Urogenital Development. Dev Biol 288, 582-594.

Wingert, R.A., Davidson, A.J., 2011. Zebrafish Nephrogenesis Involves Dynamic Spatiotemporal Expression Changes in Renal Progenitors and Essential Signals from Retinoic 
Acid and Irx3b. Developmental dynamics : an official publication of the American Association of Anatomists 240, 2011-2027.

Wingert, R.A., Selleck, R., Yu, J., Song, H., Chen, Z., Song, A., Zhou, Y., Thisse, B., Thisse, C., McMahon, A.P., Davidson, A.J., 2007. The Cdx Genes and Retinoic Acid Control the Positioning and Segmentation of the Zebrafish Pronephros. PLoS genetics 3, 1922-38.

Xu, J., Liu, H., Park, J., Lan, Y., Jiang, R., 2014. Osr1 Acts Downstream of and Interacts Synergistically with Six2 to Maintain Nephron Progenitor Cells during Kidney Organogenesis. Development 141, 1442-1452.

Xu, J., Liu, H., Chai, H., Lan, Y., Jiang R., 2016. Osr1 Interacts Synergistically with Wt1 to Regulate Kidney Organogenesis. PLoS One 11, e0159597.

Zhang, Z., Iglesias, D., Eliopoulos, N., El Kares, R., Chu, L., Romagnani, P., Goodyer, P., 2011. A Variant OSR1 Allele which Disturbs OSR1 mRNA Expression in Renal Progenitor Cells is Associated with Reduction of Newborn Kidney Size and Function. Hum Mol Genet 20, 41674174.

Zhu, X., Chen, Z., Zeng, C., Wang, L., Xu, F., Hou, Q., Liu, Z., 2016. Ultrastructural Characterization of the Pronephric Glomerulus Development in Zebrafish. Journal of Morphology 277, 1104-1112.

Zhuo, J.L., Li, X.C., 2013. Proximal Nephron. Comprehensive Physiology 3, 1079. 


\section{Figure Legends}

Figure 1: The ENU mutant ocn has a proximally abrogated pronephros due to a lesion in osr1. A. At $24 \mathrm{hpf}$, the zebrafish pronephros contains two clusters of podocytes and two nephron tubules. By $48 \mathrm{hpf}$, the pronephros is functional as the podocyte progenitors have migrated to the midline and fused. In ocn-/- mutants, podocyte progenitors $(w t 1 b)$ are reduced at both stages. The pronephric tubules $(c d h 17)$ were truncated at $24 \mathrm{hpf}$, which became more dramatic at $48 \mathrm{hpf}$. Scale bar is $50 \mu \mathrm{m}$. B. A live time course of ocn revealed pericardial edema beginning at $72 \mathrm{hpf}$, as indicated by black arrow heads. This fluid imbalance was symptomatic of organ dysfunction. Scale bar is $70 \mu \mathrm{m}$. WISH experiments to view podocytes and tubule ( $w t 1 b, c d h 17)$ were also conducted at 72 hpf. JB-4 serial sectioning was conducted on three WT and three ocn-/- embryos to examine the anterior pronephros, the location is marked by the dashed vertical line. WT siblings had an intact pronephros (dotted outline), including a glomerulus (asterisk) with two tubules. Mutant sections of this same region had no discernable blood filter or tubule structure. Scale bar is $50 \mu \mathrm{m}$. C. At 48 hpf, ocn::cdh17::GFP embryos were injected with 70 kDA rhodamine dextran (red). These embryos were assessed at 96 hpf. Nephron tubules are shown by the dotted outline. WT siblings exhibited no edema and appeared to uptake the dextran in the proximal region, as indicated by yellow coloration (inset). However, in mutants with pericardial edema and truncated tubules, there was no evidence of dextran within the tubule, suggesting that active uptake was not occurring in these mutants. Scale bar is $15 \mu \mathrm{m}$ for lateral images and $50 \mu \mathrm{m}$ for dorsal views. D. After assessment of the genetic candidates obtained via whole genome sequencing, osrl appeared to be an attractive possibility due to a $\mathrm{C}$ to $\mathrm{T} \mathrm{SNP}$ that was predicted to cause a premature stop codon. The predicted lesion (red shape) is located in exon 2 of osrl. We designed primers that flanked exon 2 (arrow heads) for Sanger sequencing. Embryos with reduced wt la WISH staining exhibited a "TGA" codon within exon 2 of osrl that is normally a "CGA" codon in WT embryos. Scale bar is $30 \mu \mathrm{m}$. E. To confirm that wtla+ podocytes were reduced in ocn-/-, FISH with wtla and wt $1 b$ was performed at $24 \mathrm{hpf}$. There were little to no double positive cells seen in genotype-confirmed mutants, whereas both clusters of $w t 1 a / b+$ podocytes were evident in WTs. Scale bar is $10 \mu \mathrm{m}$. F. The slit diaphragm marker $n p h s l$ was similarly reduced in 24 hpf ocn mutants. Scale bar is $50 \mu \mathrm{m}$. G,H. At the 15 ss, pax $2 a$ marks the developing IM, the beginning of which is shown with green arrowheads. In ocn-/-, the anterior region of pax $2 a$ is decreased. When ocn-/- was injected with osr 1 cRNA, pax $2 a$ expression was restored. Interestingly, pax $2 a$ was significantly expanded in WT embryos injected with osrl. Absolute area measurements of pax $2 a$ were taken from somites 1-5. P-values: $* * p<0.001,{ }^{*} p<0.05$, N.S. $=$ not significant. Scale bar is $50 \mu \mathrm{m}$.

Figure 2: osr1 is required to maintain and promote kidney development at the expense of hemagioblasts. A. Although pax $2 a$ is restricted in 15 ss mutants, at 7 ss, ocn-/- embryos had a pax $2 a$ domain that appeared to occupy the same domain as WT siblings. Similarly, the hemangioblast marker tall appeared mostly WT in ocn-/- at 7 ss. Scale bar is $50 \mu \mathrm{m} \mathrm{B.} \mathrm{FISH} \mathrm{with}$ probes for pax $2 a$ (green) and tall (red) and ICC using anti-caspase-3 (white) to mark apoptotic cells was conducted at 7 ss and 15 ss. The number of fragments in the combined pax $2 a$ and tall fields from somites 1-5 were increased in mutants at $7 \mathrm{ss}$, but not at 15 ss. Scale bar is $35 \mu \mathrm{m}$ C,D,E. At $10 \mathrm{ss}$, little to no caspase- 3 fragments were seen in pax $2 a$ or tall domains from somites $1-5$, but a significant number were seen in osrl morphants and ocn-/-. The tall domain was also expanded in both loss of function models. Scale bar is $35 \mu \mathrm{m}$. F,G. ICC with the proliferative cell marker anti-pH3 was also conducted. Despite the expansion in tall in osr l deficient models, there was no significant change in the number of proliferating cells. Scale bar is $10 \mu \mathrm{m}$. H,I. FISH experiments were conducted to assess changes in apoptosis in wt $1 a+$ pax $2 a+$ podocyte progenitors. 
There was a significant increase in the number of apoptotic fragments within this field in mutants compared to WT siblings. Scale bar is $10 \mu \mathrm{m}$. A minimum of three individuals were assessed for each group across experiments. Photos are max intensity projections from z-stacks, and each side of mesoderm was quantified individually. P-values: ${ }^{* *} \mathrm{p}<0.001$, N.S. $=$ not significant.

Figure 3: osr1 is required for continued development of kidney lineages. A,B. Embryos from ocn incrosses were injected at the 1-cell stage with 50 pgs of osr 1 cRNA and examined. At 15 ss, podocytes $(w t 1 b)$ were robustly rescued in $o c n-/-$. C,D. However, by 22 ss, podocytes were only partially rescued in ocn-/- injected embryos, while tubule length in the same animals were not significantly different from WTs, as shown by the green arrowheads. E,F,G. Truncated tubule was still able to be rescued in mutants at $24 \mathrm{hpf}$. Further, overexpression of osrl induced ectopic tubule formation (green arrowheads). H. A rescue time course was conducted with osrl MO and osrl cRNA to determine when the osrl cRNA dosage became insufficient to rescue. While there was a $90 \%$ rescue rate at $24 \mathrm{hpf}$, by $48 \mathrm{hpf}$, this rate had dropped to $20 \%$. This indicated that continued osrl is needed for normal tubule development. P-values: ${ }^{*} \mathrm{p}<0.001, \mathrm{~N} . \mathrm{S}$. = not significant. For tubule rescue at $24 \mathrm{hpf}, \mathrm{p}$-values were obtained from arcsin transformed kidney to body percentage calculations for each group. Scale bar is $50 \mu \mathrm{m}$ for all images.

Figure 4: wnt $2 b \boldsymbol{a}$ is a podocyte marker and regulator. A. wnt $2 b a$ is expressed in bilateral stripes as early as 13 ss. Scale bar is $30 \mu \mathrm{m}$. B. wnt $2 b a$ (red) is expressed within the anteriormost region of the IM, as shown by co-localization with pax $2 a$ (green). White box denotes area of colocalization, which is magnified in bottom panel. DAPI (blue) marks nuclei. Scale bar is $15 \mu \mathrm{m}$. C. $w n t 2 b a$ (red) also colocalized with the podocyte marker $w t 1 b$ at $24 \mathrm{hpf}$, though at this time point it was also expressed in putative neck segment domain. By $48 \mathrm{hpf}$, the wnt $2 \mathrm{ba}$ domain was specified to the podocytes. Scale bar is $30 \mu \mathrm{m}$. D-H. Podocyte area and cell number was assessed in wnt $2 b a$ morpholino-injected animals and determined to be reduced compared to WT controls. Both $w t 1 b$ and $n p h s 1$ showed a significant decrease in domain area in wnt $2 b a$ morphants compared to WT embryos. I,J. FISH with wt $1 a$ and $w t 1 b$ was performed at $24 \mathrm{hpf}$. There was a significant area reduction in podocyte domain seen in wnt $2 b a$ morphants compared to WTs. Scale bar is 10 $\mu \mathrm{m}$. P-values: ${ }^{*} \mathrm{p}<0.001,{ }^{*} \mathrm{p}<0.05, \mathrm{~N} . \mathrm{S}$. $=$ not significant. Scale bar is $30 \mu \mathrm{m}$.

Figure 5: wnt $2 b a$ is sufficient for podocyte development downstream of osr 1. A. When wnt $2 b a$ was assessed at $15 \mathrm{ss}$, in ocn-/- and WT siblings, it was evident that staining was reduced in mutants. By $24 \mathrm{hpf}$, wnt $2 b a$ staining was almost completely absent, tracking with the loss of other podocyte markers in $o c n-/-$. Scale bar is $30 \mu \mathrm{m}$. B. FISH experiments showed that $o s r l$ and wnt $2 b a$ colocalized in cells at $22 \mathrm{ss}$. C-H. Embryos were injected with osrl MO and/or wnt $2 b a$ cRNA at the one cell stage and podocytes and the developing slit diaphragm were visualized at $24 \mathrm{hpf}$ using $w t 1 b$ and $n p h s 1$, respectively. Embryos injected with osrl MO alone showed few podocyte or slit diaphragm cells, while embryos injected with wnt $2 b a \mathrm{cRNA}$ alone had an increased podocyte area. Co-injected embryos had a partial rescue of podocytes, indicating that wnt $2 b a$ is a downstream factor in the podocyte pathway. A minimum of 5 individuals were imaged for quantification. Pvalues: $* * p<0.001,{ }^{*} \mathrm{p}<0.05$, N.S. $=$ not significant. Scale bar is $30 \mu \mathrm{m}$.

Figure 6: Acting in opposition to osr1, hand2 inhibits wnt2ba-driven podocyte development A,B,C. Embryos from ocn incrosses were injected with hand2 MO. Podocytes area and cell number were partially rescued in ocn-/- injected embryos, and were expanded in WT injected siblings. This signified that hand 2 suppresses podocyte formation. D,E. Similarly, wnt $2 b a$ expression was rescued in osr l/hand 2 morphants compared to uninjected WT controls. Injection 
of hand2 $\mathrm{MO}$ alone led to a significant increase in the wnt2ba domain. All images were at $24 \mathrm{hpf}$, scale bar is $30 \mu \mathrm{m}$. A minimum of 5 individuals were imaged for quantification. P-values: ${ }^{*} \mathrm{p}<0.001,{ }^{*} \mathrm{p}<0.05, \mathrm{NS}=$ not significant. F. osrl promotes podocyte and kidney lineages and suppress blood and vasculature, while hand 2 acts in opposition. Imbalance of either of these factors leads to changes in mesodermal fates. Podocyte development is one example of a mesodermal fate that is altered by imbalance of osr $1 /$ hand2. This is because the downstream factor $w n t 2 b a$ is decreased without osr 1 , yet increased in the absence of hand2. wnt $2 b a$ endorses the podocyte factors $w t 1 a / b$, which have been shown to be required for formation of podocytes and the slit diaphragm (nphs1).

\section{Supplemental Figure Legends}

Figure 1, Supplement 1: Additional ocn phenotypes. A. Conducting absolute length measurements of $c d h 17$ tubule revealed that ocn have significantly shorter nephrons tubules at 24 hpf and $48 \mathrm{hpf}$ compared to WT siblings. B. A live time course of ocn-/- revealed pericardial edema beginning at $72 \mathrm{hpf}$, as indicated by black arrow heads. Scale bar is $70 \mu \mathrm{m}$. C. ocn+/- were crossed with a cdh17::GFP transgenic line to create ocn carriers with GFP-labeled pronephric tubules. Pictured here are 72 hpf cdh17::GFP embryos and F2 ocn-/-::cdh17::GFP embryos. White arrowheads demarcate the start and terminus of the pronephros, and it is evident that mutants have a shorter pronephros. Scale bar is $30 \mu \mathrm{m}$. D. Similarly to WISH results with $c d h 17$ and $w t 1 b$, IF revealed that ocn-/- embryos had a truncation in NaKATPase+ tubule and absence of aPKC + glomerulus. E. The acidic stain, alcian blue, was used to show cartilage in developing ocn-/- and WT siblings at $96 \mathrm{hpf}$. Lateral and ventral views showed that the jaw developed in an improper orientation with unfused Meckel's cartilage (box). Scale bar is $30 \mu \mathrm{m}$. Pectoral fins in ocn-/mutants were also malformed. F,G. The pectoral fins arise from the fin buds (white dotted area), which are marked by mecom at $24 \mathrm{hpf}$. Fin bud area measurements were decreased in mutants, which could be genotyped by the absence of $w t 1 b+$ podocytes. Scale bar is $30 \mu \mathrm{m}$. P-values: $* * \mathrm{p}<0.001$.

Figure 1, Supplement 2: A genetic lesion in the osr1 transcription factor would result in truncated osr1 protein in the ocn mutant line A. Whole genome sequencing was conducted on 24 hpf ocn mutants and WT siblings. SNPtrack analysis indicated that the genetic lesion responsible for the ocn-/- phenotypes was most likely located on chromosome 13. Specifically, a $\mathrm{C}$ to $\mathrm{T}$ missense mutation in exon 2 of osrl would cause a premature stop codon. B. Zebrafish, mouse and human OSR1 protein contains three zinc-finger binding domains. The predicted SNP would result in a substitution from an arginine to a stop codon before the transcription of the zincfinger binding domains, which are each 100\% conserved across zebrafish, mice and human OSR1 protein.

Figure 1, Supplement 3: Verification of osr1 splice-blocking morpholino phenotypes. A,B. A previously published morpholino was obtained to block splicing activity in exon 2 of osr 1 . Primers flanking exon 2 (arrowheads) were used to conduct RT-PCR to assess splicing activity in morphants at $24 \mathrm{hpf}$. In WTs with correct splicing, a 1000 bp product is obtained. In osrl morphants, intron 2 fails to be spliced out the gene, leading to a $631 \mathrm{bp}$ increase in product. Further, the intronic sequence retained in osr 1 morphants contains in-frame stop codons. C. osr 1 morphants had dramatically decreased podocytes and a cropped pronephric tubule which resembled both previously published results and ocn-/-. Scale bar is $30 \mu \mathrm{m}$. 
Figure 2, Supplement 1: Additional analysis of cell dynamics in osr1 deficient models. A. FISH experiments were conducted on young embryos (11 ss $=15 \mathrm{hpf})$ and flatmounted to be imaged. Probes for pax $2 a$ (green) were used to mark IM, and tall (red), to mark hemangioblasts. DAPI (blue) marks nuclei and was used to distinguish cellular features such as muscular units known as somites. Counting somites allowed for accurate staging of embryos and a consistent location for tissue assessment. Areas and cell counts of mesodermal tissues were assessed from somites 1-5, as shown in the boxed area. B. There was a significant increase in caspase-3+ fragments in the tal1/pax $2 a$ area of interest in ocn-/- compared to siblings at 7 ss. However, by 15 ss, the number of caspase-3+ fragments in ocn-/- had returned to a WT level. C. Compared to WT sibings, the tall domain steadily increased in ocn-/- from 7 to $15 \mathrm{ss}$. In contrast, the pax $2 a$ domain only became significantly smaller in ocn-/- mutants at the 15 ss. D,E. There was no significant change in the number of $\mathrm{pH} 3+$ cells in osr 1 morphants in the tall domain between somites 1-5 at the 8 ss.

Figure 3, Supplement 1: osr1 cRNA injection additional analysis. A. ocn-/- and WT sibling embryos injected with osrl cRNA had significantly shorter body lengths than uninjected ocn-/mutants and siblings. P-values: $* * p<0.001$, N.S. = not significant. P-values were obtained by arcsin transforming percentages to normalize data. B. Time course of $c d h 17$ expression in WT and osrl MO injected animals. Scale bar is $20 \mu \mathrm{m}$.

Figure 4, Supplement 1: wnt2ba colocalizes with early, developing podocytes A,B. Lateral views of FISH experiments reveal that wnt $2 b a$ is present in developing $w t 1 a$ and $w t 1 b$ podocytes at $15-17$ ss and 22-24 ss. Scale bar is $10 \mu \mathrm{m}$.

Figure 4, Supplement 2: wnt2bb does not colocalize with podocytes. A. Lateral views of WT embryos stained using FISH reveal that $w n t 2 b b$ is expressed anterior to $w t 1 b$ podocytes at $24 \mathrm{hpf}$. Scale bar is $50 \mu \mathrm{m}$.

Figure 4, Supplement 3: Assesment of wnt2ba MO through RT-PCR analysis. A. The gene wnt $2 b a$ contains 5 exons. A morpholino was utilized to block splicing activity in intron 1 . B. In WT embryos, correct splicing activity occurs to splice exon 1 and 2 together, resulting in a $\sim 300$ bp product as seen with F1/R1 primers. This product is greatly diminished in wnt $2 b a$ morphants, suggesting that correct splicing activity is limited. C. Primers were utilized that flanked the end of intron 1 and the middle of exon 2 in wnt2ba (F2/R2). In wnt2ba morphants, a strong band was present that indicated the presence of un-spliced, intronic sequence D. Primers flanking the entire ORF (F1/R2) were used as an additional metric to gauge splicing action. WTs exhibit a $1000 \mathrm{bp}$ band which represents the wnt2ba ORF. wnt2ba morphants do not have this band, which further suggests that splicing activity is impaired. E. An ATG MO was used to knockdown wnt $2 b a$. While podocytes were reduced using this reagent, the pronephric tubule was not affected. The start of the tubule is shown using green arrowheads. Scale bar is $50 \mu \mathrm{m}$.

Figure 4, Supplement 4: wnt2ba knockdown causes decreased podocytes at 22 ss. A-D. wnt $2 b a$ was knocked down using a splice-blocking MO and podocytes were examined by conducting WISH on 15 ss and 22 ss embryos using the marker $w t 1 b$. Anti-pH3 was used on these samples to assess proliferating cells. Podocyte area and cell counts were not different between morphants and WTs at $15 \mathrm{ss}$, though there was a significant decrease in podocyte cell number and domain area at 
$22 \mathrm{ss}$ in morphants. However, there were no changes in the number of $\mathrm{pH} 3+$ cells in the $w t 1 b$ area at either time point. Scale bar is $40 \mu \mathrm{m}$.

Figure 5, Supplement 1: Additional stages of wnt2ba and osr1 co-localization. A. In addition to $22 \mathrm{ss}$, it was also observed that $o s r l$ and wnt $2 b a$ colocalized in a subset of presumptive IM at 15-17 ss. Scale bar is $10 \mu \mathrm{m}$.

Figure 6, Supplement 1: hand2 MO replicates previous studies. A. The developing heart tube can be visualized with myl7 at 22 ss. Embryos injected with hand2 MO display cardia bifida, or a separation of heart precursors. 
bioRxiv preprint doi: https://doi.org/10.1101/2020.12.21.423845; this version posted December 22,2020 . The copyright holder for this preprint

(which was not certified by peer review) is the author/funder, who has granted bioRxiv a license to display the preprint in perpetuity. It is made available under aCC-BY-NC-ND 4.0 International license.

\section{Figure 1}

A

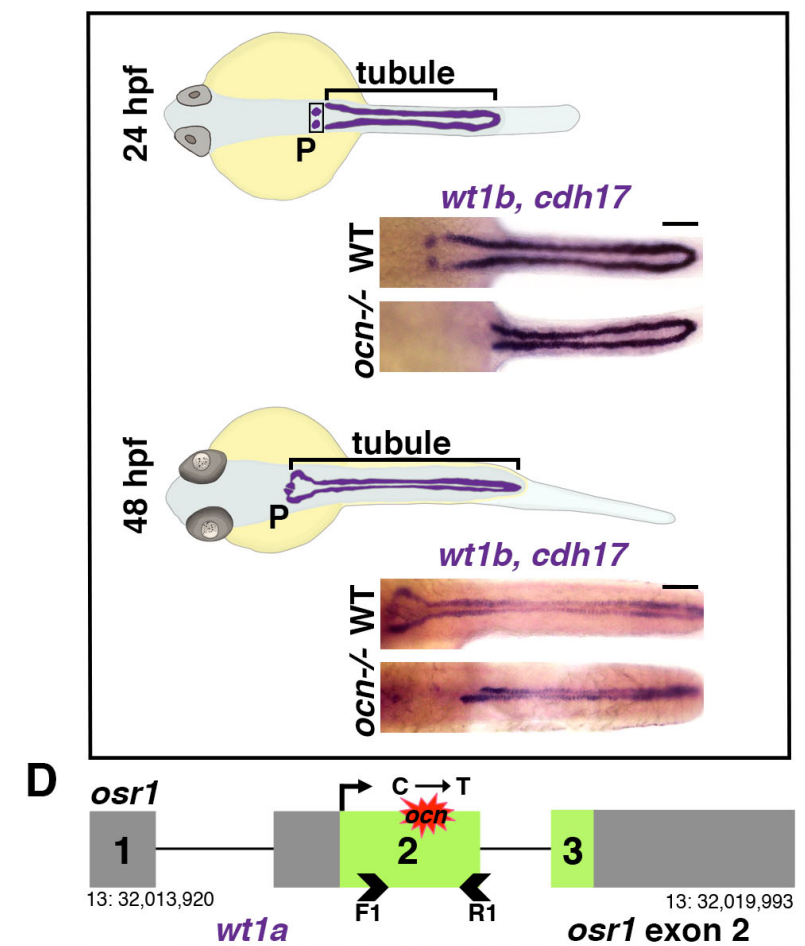

B

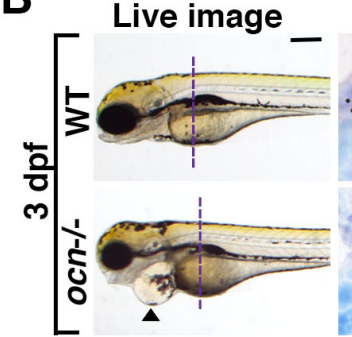

C

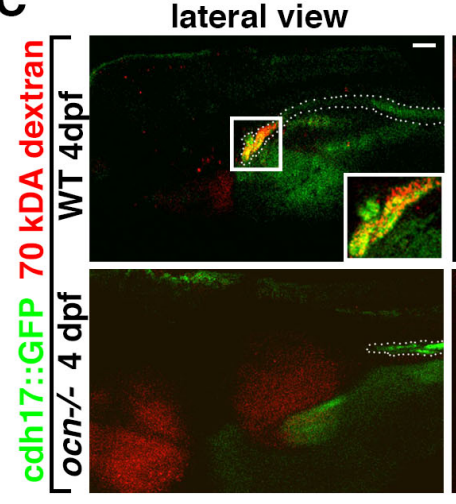

E

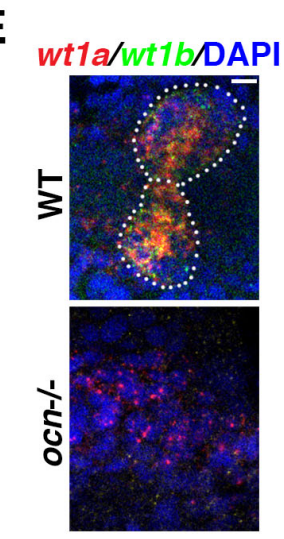

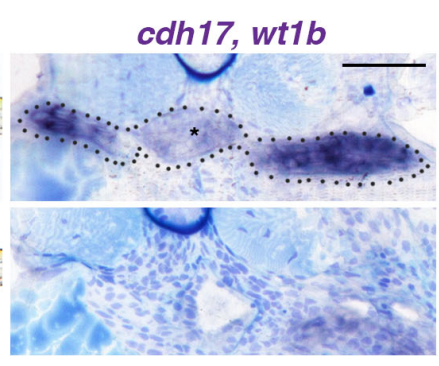

dorsal view

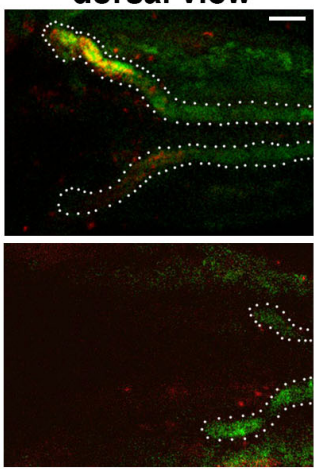

$\mathbf{F}$

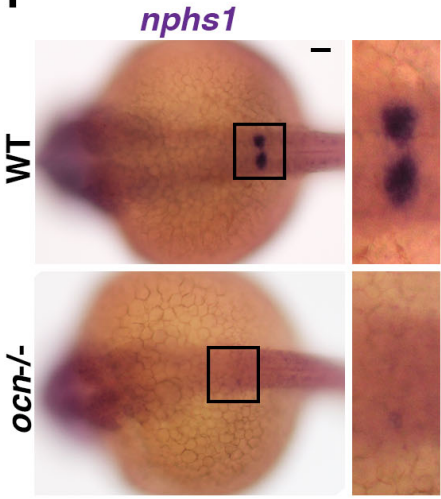

G

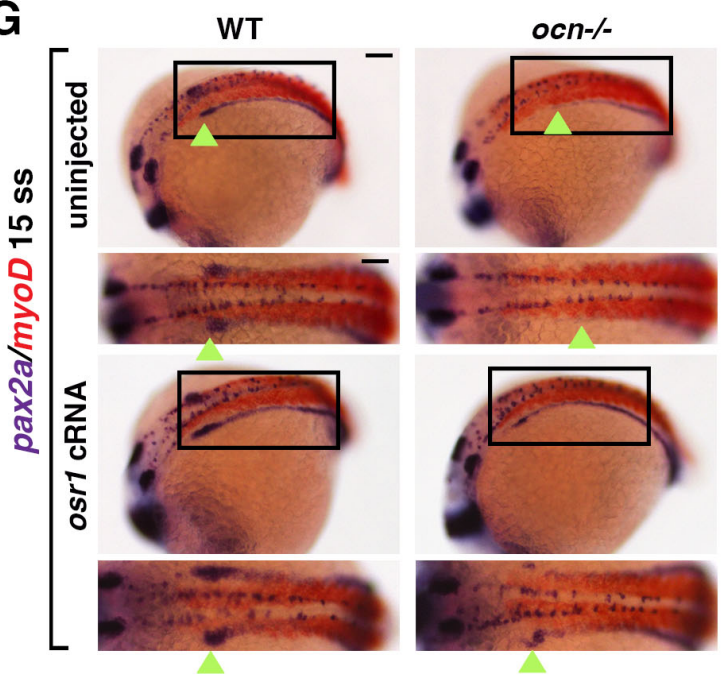

H

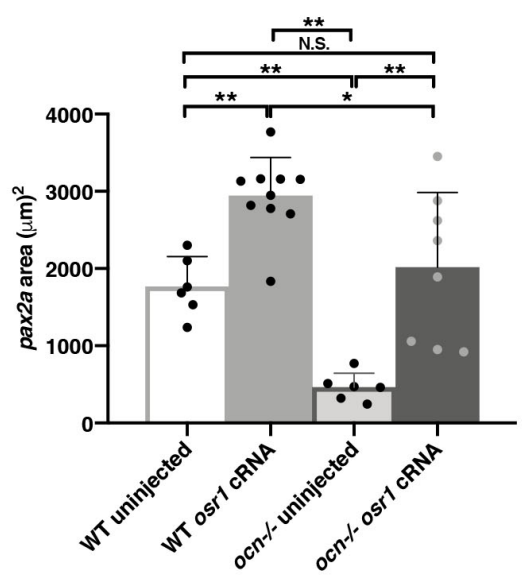


bioRxiv preprint doi: https://doi.org/10.1101/2020.12.21.423845; this version posted December 22, 2020. The copyright holder for this preprint (which was not certified by peer review) is the author/funder, who has granted bioRxiv a license to display the preprint in perpetuity. It is made available under aCC-BY-NC-ND 4.0 International license.

\section{Figure 1, Supplement 1}

A

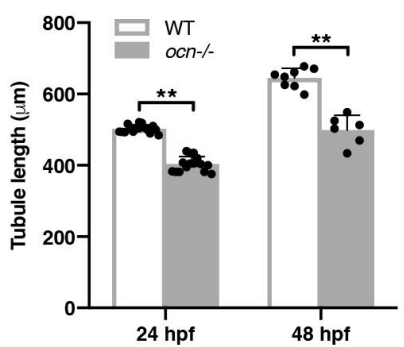

C

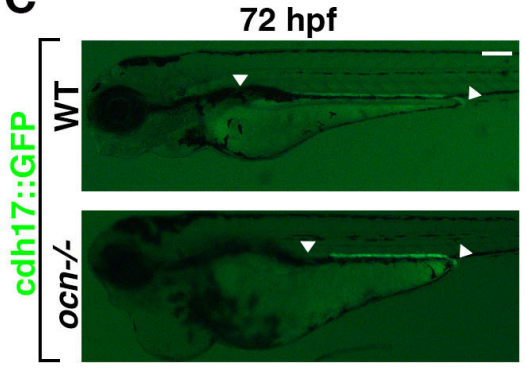

E

Alcian blue $\mathbf{9 6} \mathrm{hpf}$ Lateral

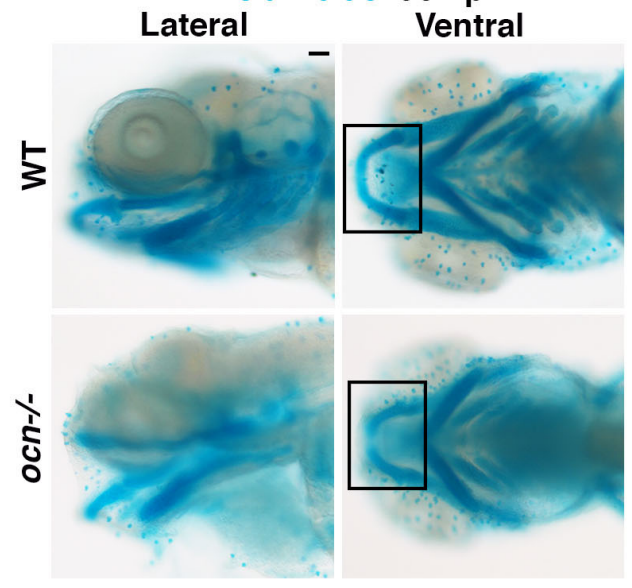

B

D
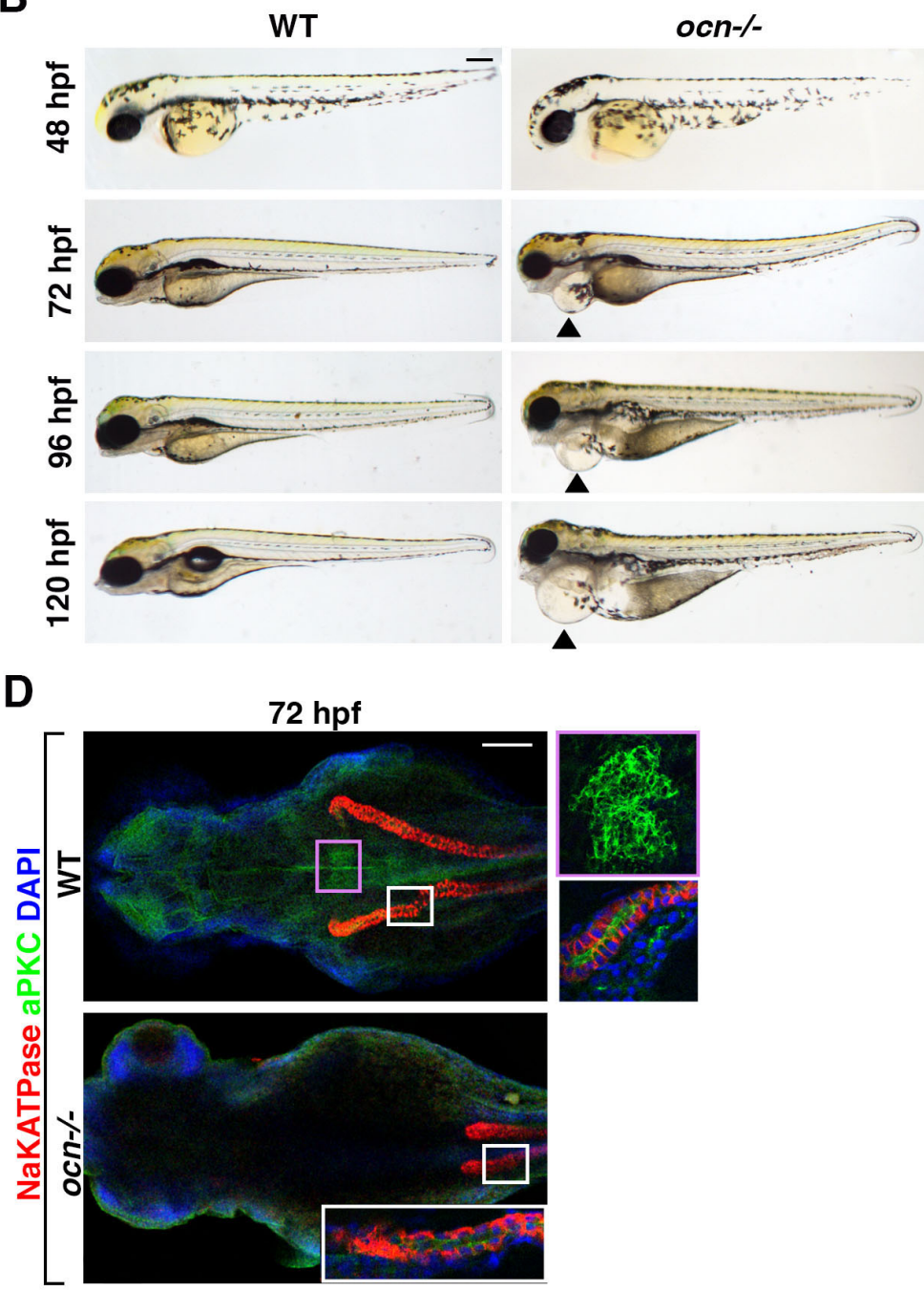

E'

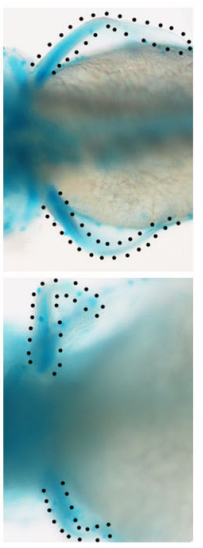

$\mathbf{F}$

mecom, wt1b $24 \mathrm{hpf}$

G

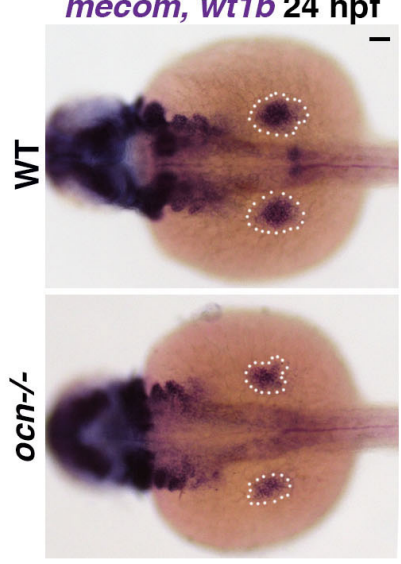

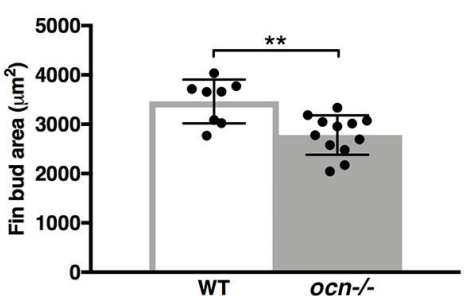


bioRxiv preprint doi: https://doi.org/10.1101/2020.12.21.423845; this version posted December 22, 2020. The copyright holder for this preprint (which was not certified by peer review) is the author/funder, who has granted bioRxiv a license to display the preprint in perpetuity. It is made available under aCC-BY-NC-ND 4.0 International license.

Figure 1, Supplement 2

A

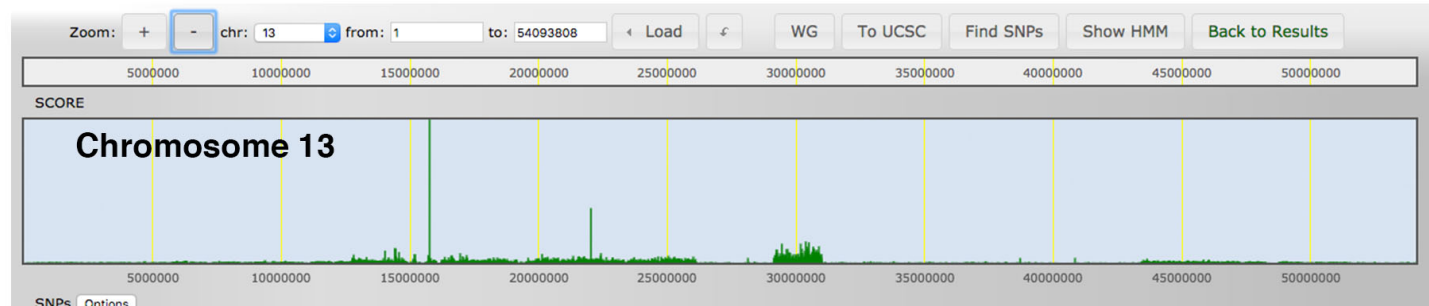

\section{osr1 exon 2}

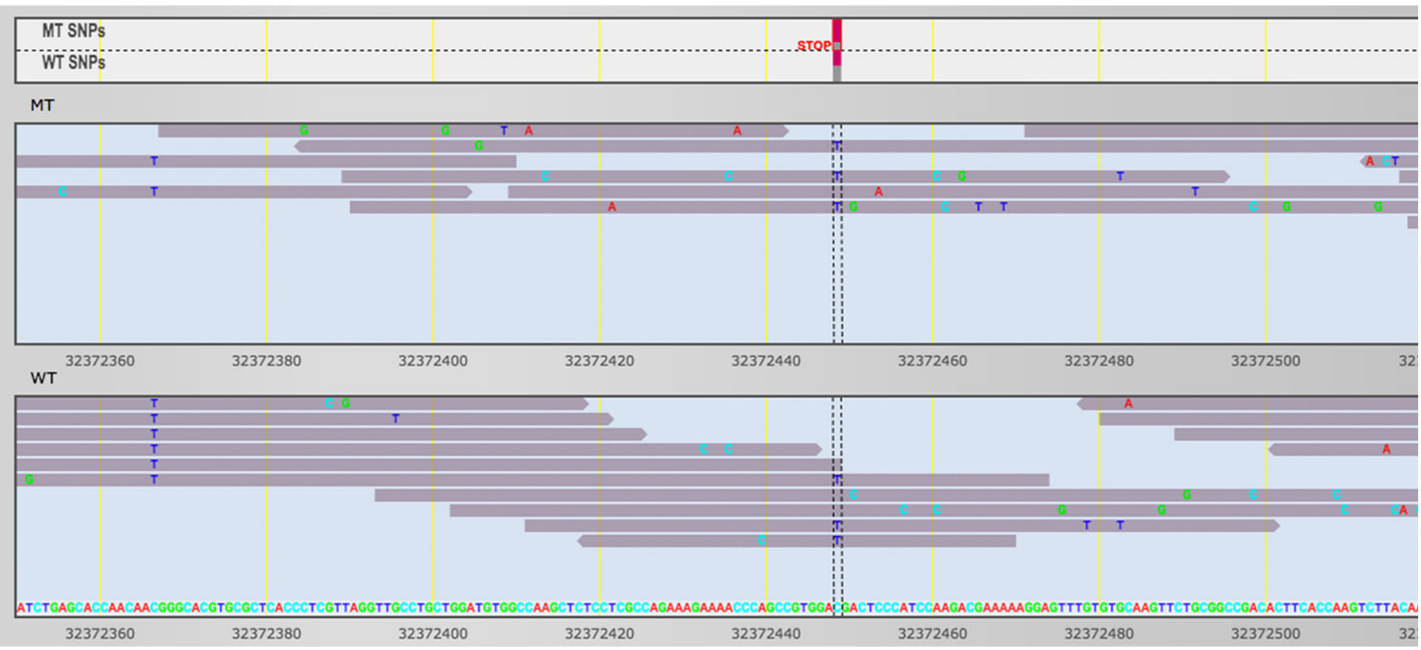

B

\begin{tabular}{|c|c|c|}
\hline Human & 1 & $\begin{array}{l}\text { MGSKTLPAPVPIHPSLQLTNYSFLQAVNGLPTVPSDHLPNLYGFSALI } \\
\text { MGSKTIPAPVPIHPSLOITNYSFLOAVNGLPTVPSDLPNLYGFSALF }\end{array}$ \\
\hline Mouse & 1 & MGSKTLPAPVPIHPSLQLTNYSFLQAVNGLPTVPSDHLPNLYGFSALH \\
\hline Zebrafish & 1 & MGSKTLPAF \\
\hline Guman & 51 & HLHQWTLGYPAMHLPRSSFSKVPGTVSSLVDARFQLPAFPWFPHVIQ \\
\hline Mous & 51 & HLHQWTLGYPAMHLPF \\
\hline Zebrafish & 50 & ¿VDAREPLPSIPLFPHLVQP \\
\hline man & 00 & PEITAGGSVPALKTKPF \\
\hline Mou & 100 & PEITAGGSGAALKTKPRFDFANLALAATQEDPI \\
\hline Zebr & 96 & 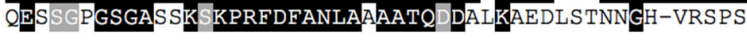 \\
\hline & & $\mathbf{v}$ \\
\hline uman & 47 & LGALLDVTKL-SPEKKPTRGRL \\
\hline Mou: & 47 & LGALLDVTKL-SPEKKPTRGRL \\
\hline Zebrafish & 145 & LGCLLDVAKLSSPERKPRGRLF \\
\hline & & \\
\hline Huma & 196 & THTDERPYTCDICHKA \\
\hline & 96 & IRYIHSKEKPFKCQECGKGFCQS \\
\hline Zebrafish & 195 & THTDERPYTCDICHKAFRRQDHLRDHRYIHSKEKPFKCQECGKGFCQS \\
\hline & & Zinc finger 1 \\
\hline & 246 & Zinc finger 2 \\
\hline Mouse & 246 & LAVHKTLHSQVKELKTSKIKC \\
\hline Zebrafish & 245 & LAVHKTLHMQVKELKPAKIK- \\
\hline
\end{tabular}


bioRxiv preprint doi: https://doi.org/10.1101/2020.12.21.423845; this version posted December 22, 2020. The copyright holder for this preprint (which was not certified by peer review) is the author/funder, who has granted bioRxiv a license to display the preprint in perpetuity. It is made available under aCC-BY-NC-ND 4.0 International license.

\section{Figure 1, Supplement 3}

A

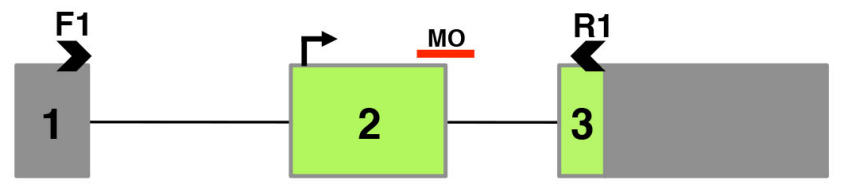

osr1 gene map

B
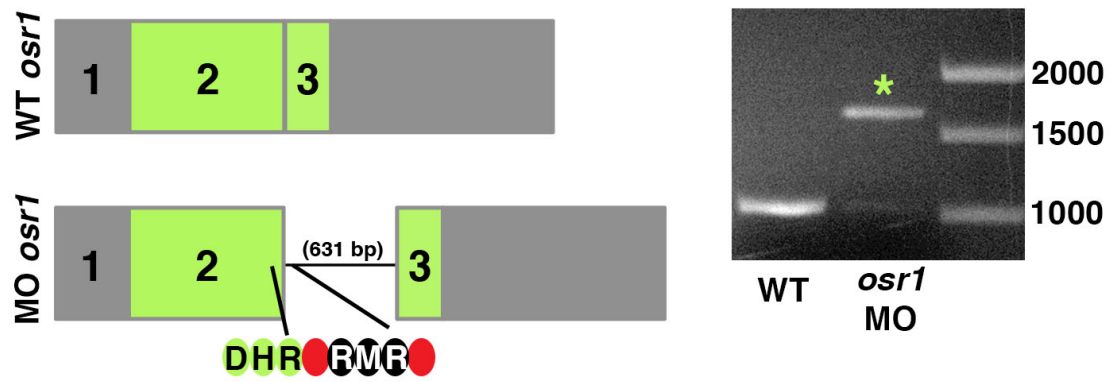

C cdh17, wt1b

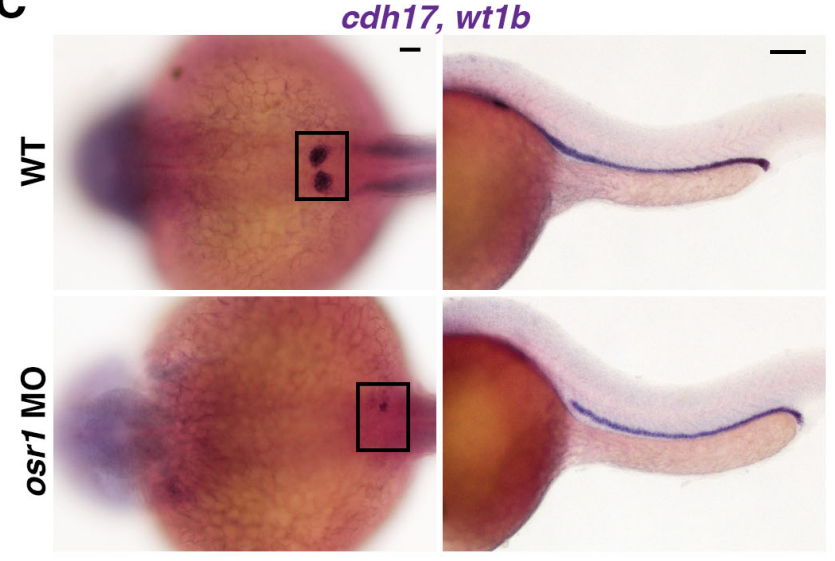


bioRxiv preprint doi: https://doi.org/10.1101/2020.12.21.423845; this version posted December 22, 2020. The copyright holder for this preprint

(which was not certified by peer review) is the author/funder, who has granted bioRxiv a license to display the preprint in perpetuity. It is made available under aCC-BY-NC-ND 4.0 International license.

\section{Figure 2}

A

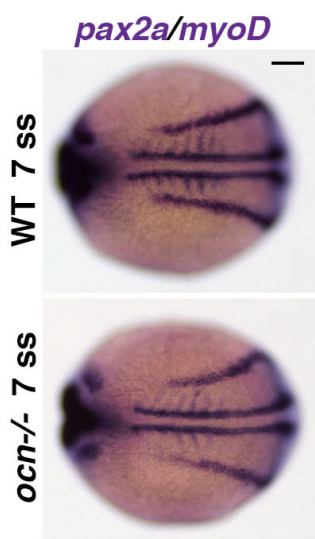

tal1

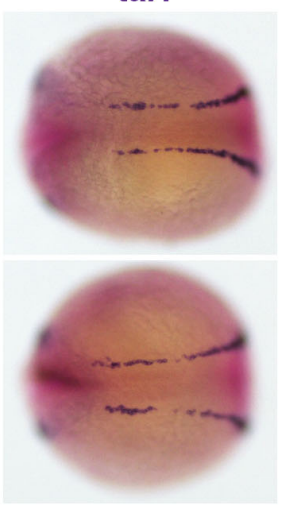

B

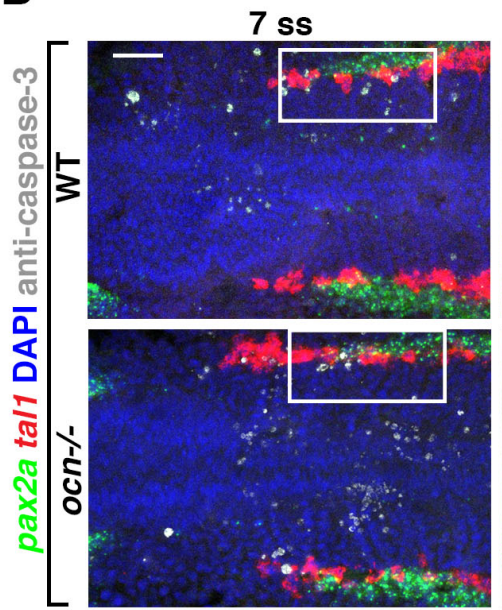

15 ss
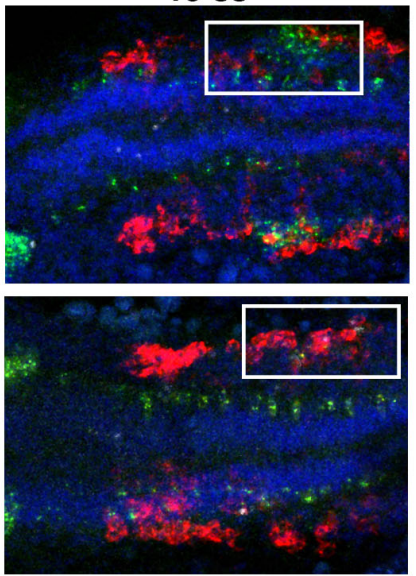

C
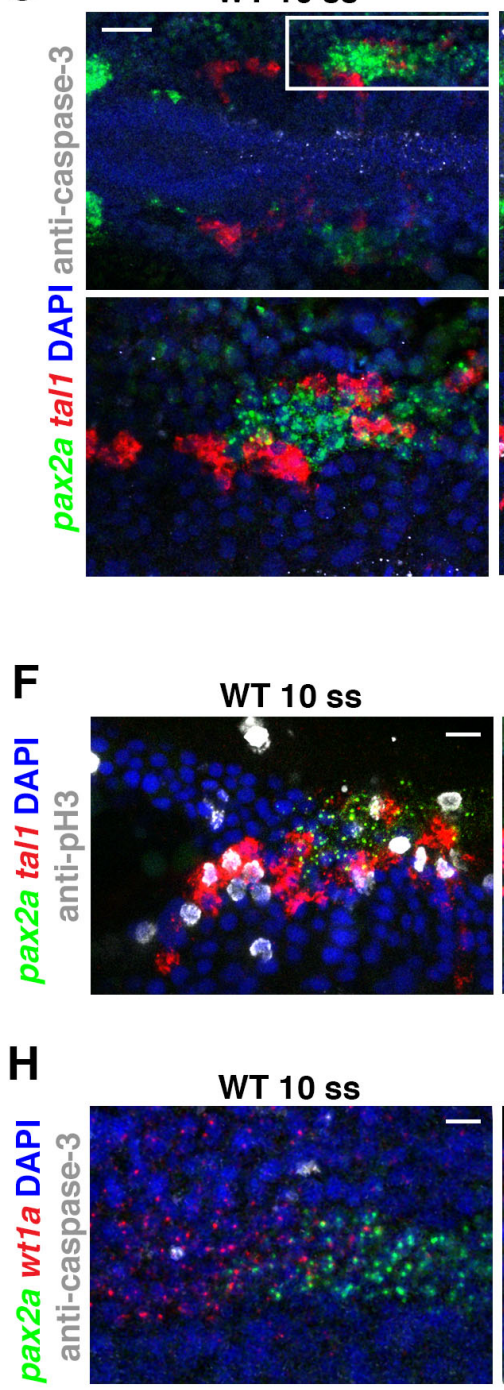

osr1 MO 10 ss
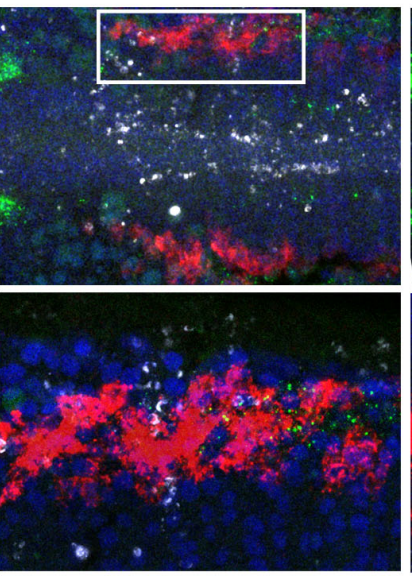

osr1 MO 10 ss

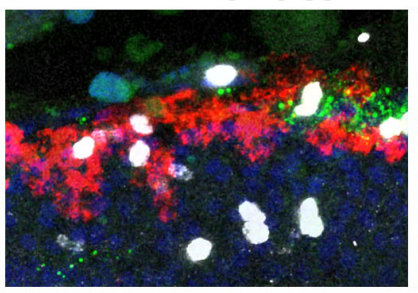

osr1 MO 10 ss

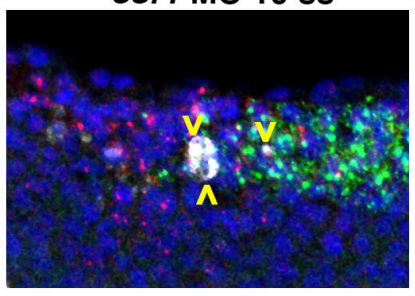

ocn-/- 10 ss
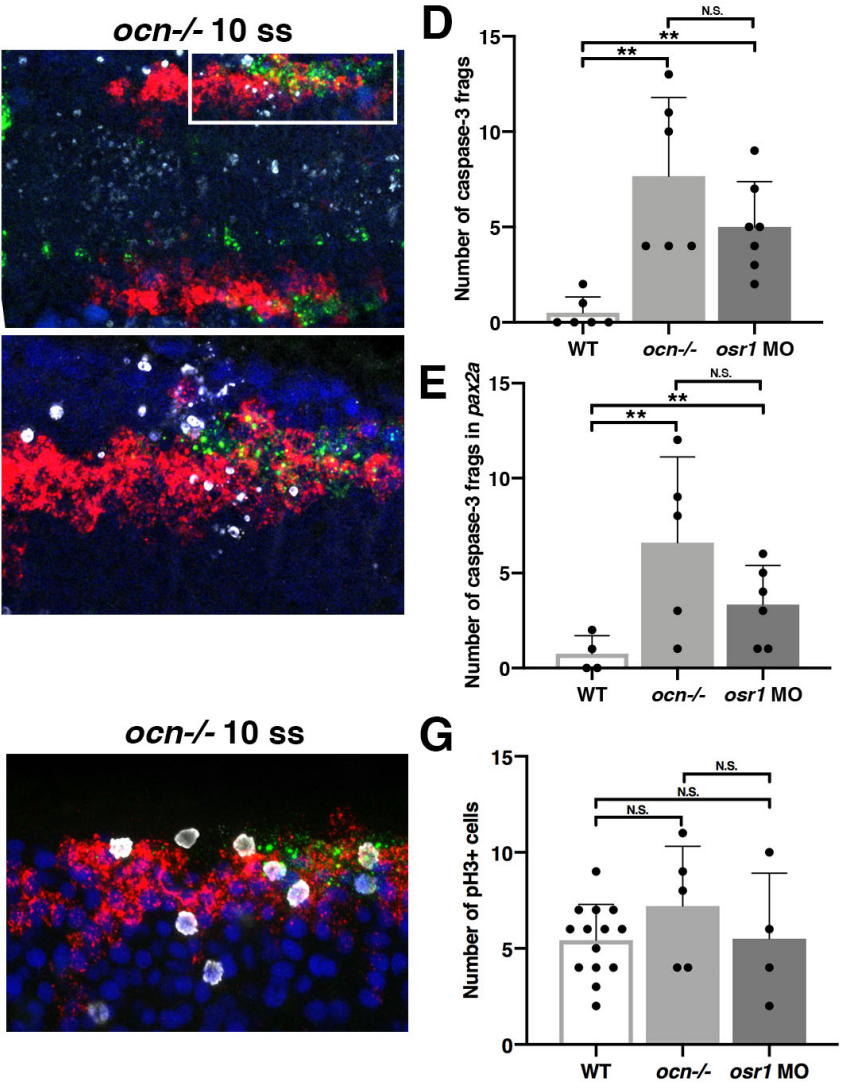
bioRxiv preprint doi: https://doi.org/10.1101/2020.12.21.423845; this version posted December 22, 2020. The copyright holder for this preprint (which was not certified by peer review) is the author/funder, who has granted bioRxiv a license to display the preprint in perpetuity. It is made available under aCC-BY-NC-ND 4.0 International license.

\section{Figure 2, Supplement 1}

A

Zebrafish flatmount

(11 ss)

pax2a tal1 DAPI

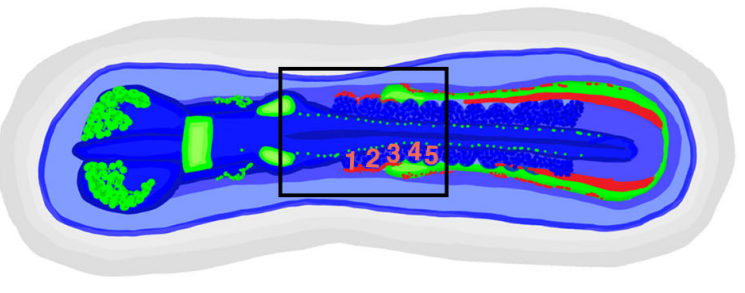

B

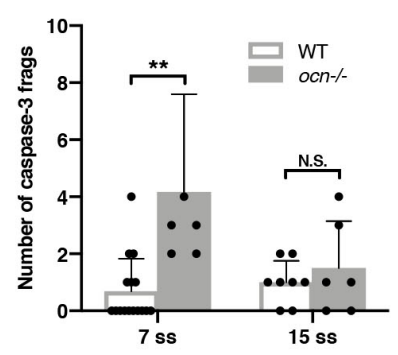

D

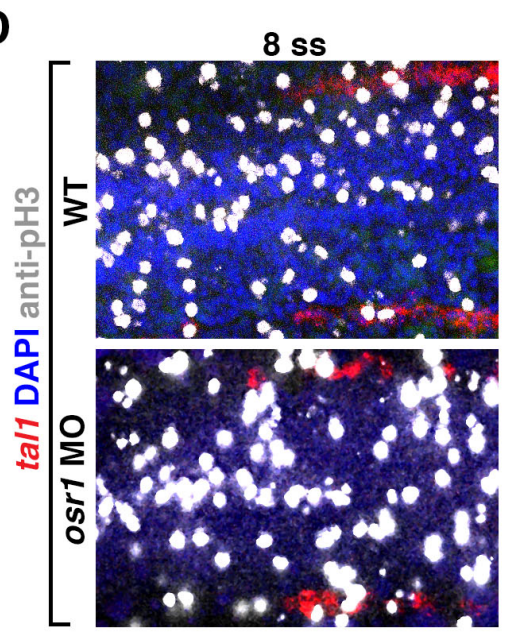

C

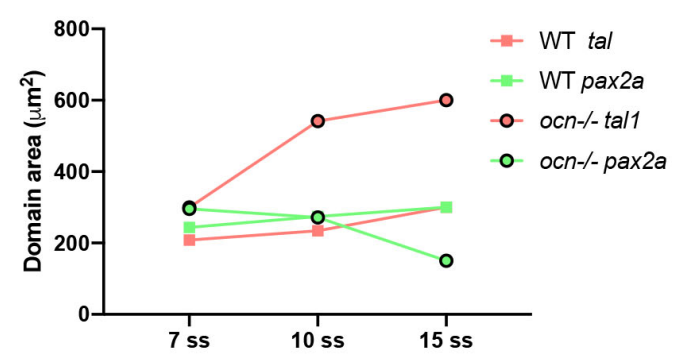

E

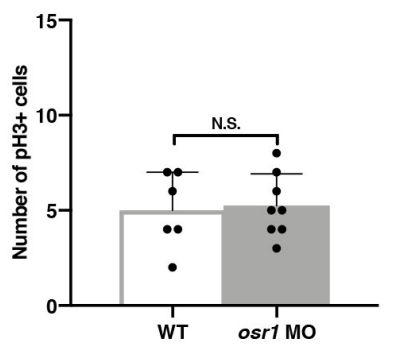


bioRxiv preprint doi: https://doi.org/10.1101/2020.12.21.423845; this version posted December 22, 2020. The copyright holder for this preprint (which was not certified by peer review) is the author/funder, who has granted bioRxiv a license to display the preprint in perpetuity. It is made available under aCC-BY-NC-ND 4.0 International license.

\section{Figure 3}

A

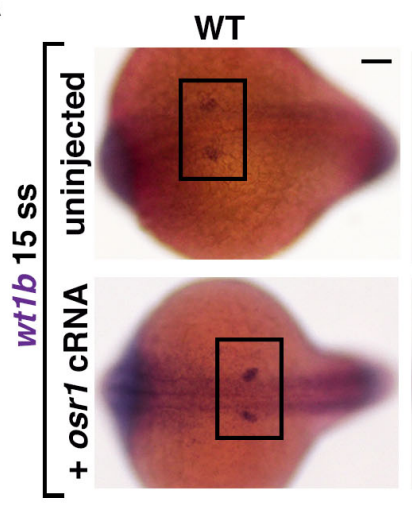

C

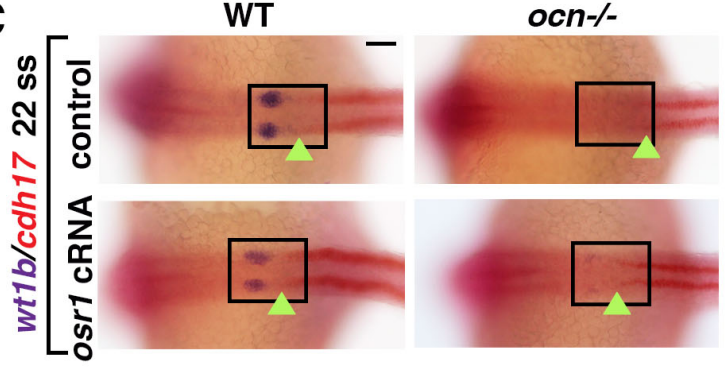

E

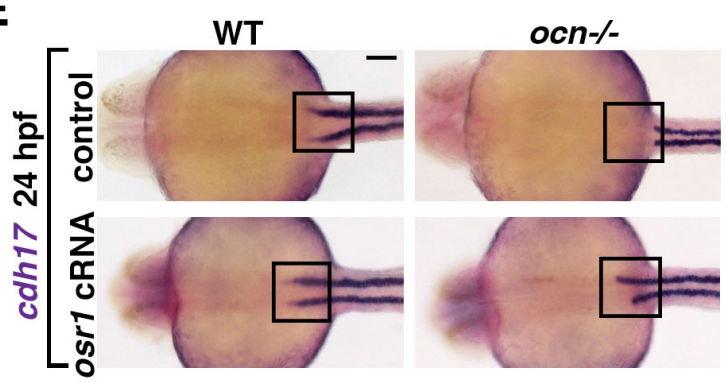

$\mathbf{F}$

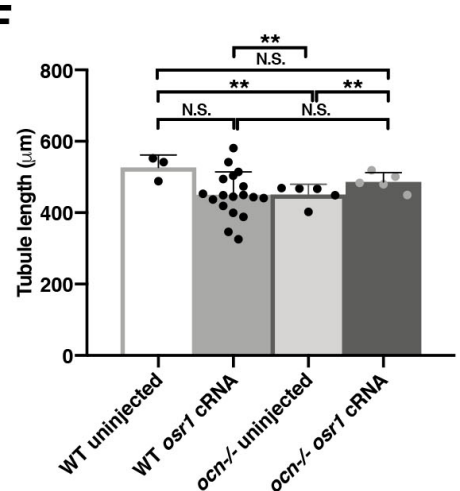

D

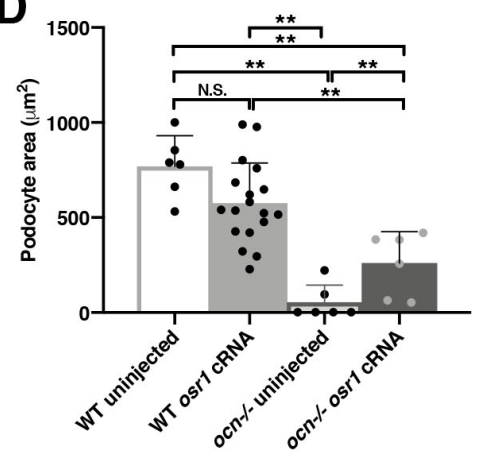

B

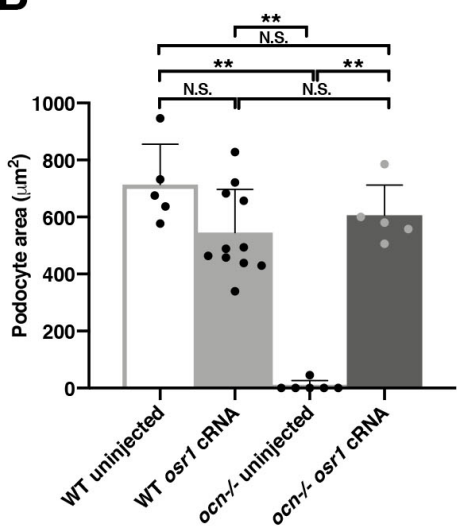

G

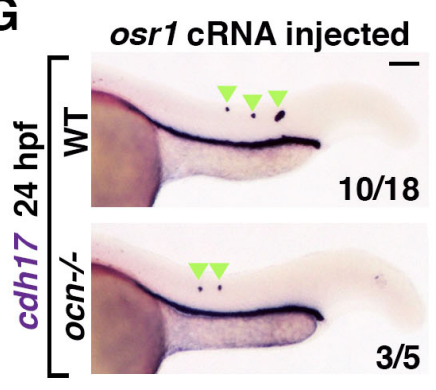

H

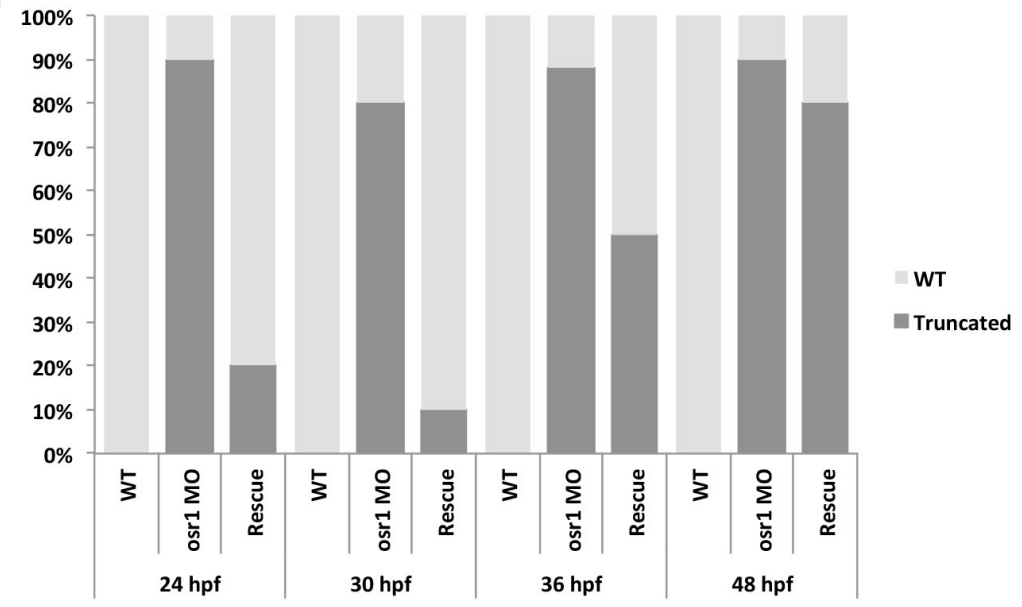


bioRxiv preprint doi: https://doi.org/10.1101/2020.12.21.423845; this version posted December 22, 2020. The copyright holder for this preprint (which was not certified by peer review) is the author/funder, who has granted bioRxiv a license to display the preprint in perpetuity. It is made available under aCC-BY-NC-ND 4.0 International license.

\section{Figure 3, Supplement 1}

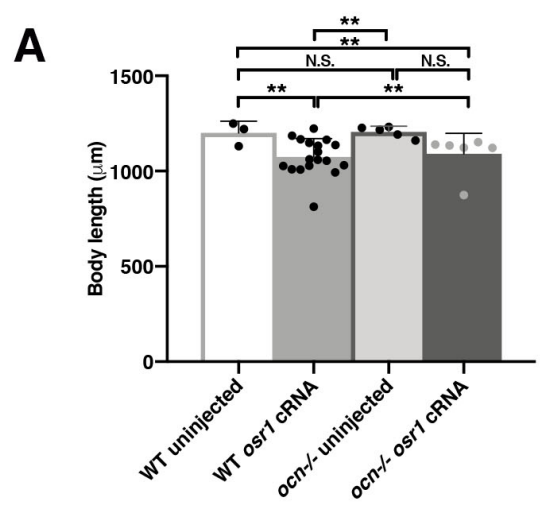

B

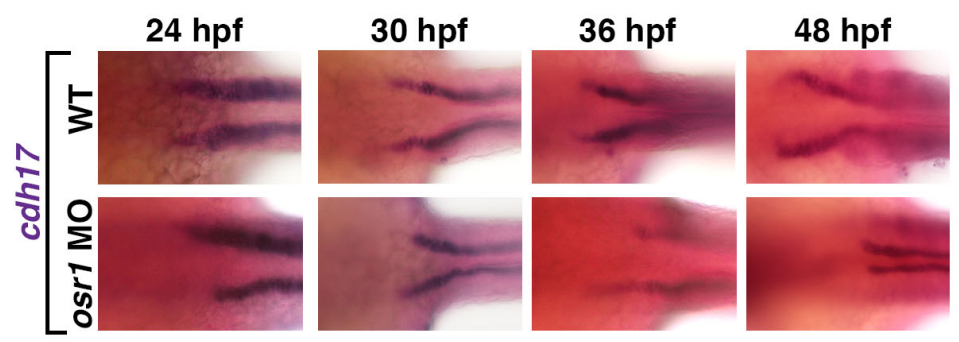


bioRxiv preprint doi: https://doi.org/10.1101/2020.12.21.423845; this version posted December 22, 2020. The copyright holder for this preprint (which was not certified by peer review) is the author/funder, who has granted bioRxiv a license to display the preprint in perpetuity. It is made available under aCC-BY-NC-ND 4.0 International license.

\section{Figure 4}

A

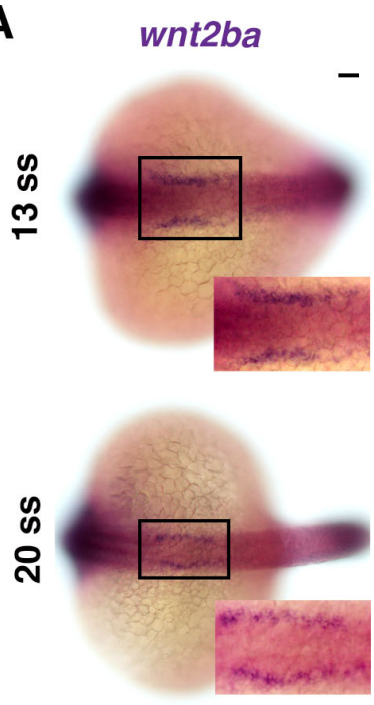

D

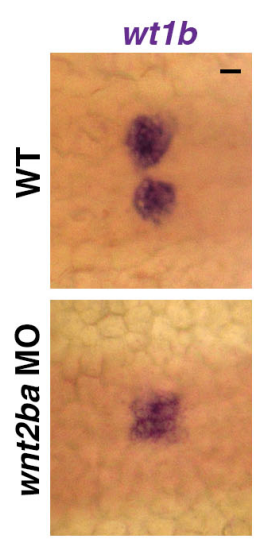

I

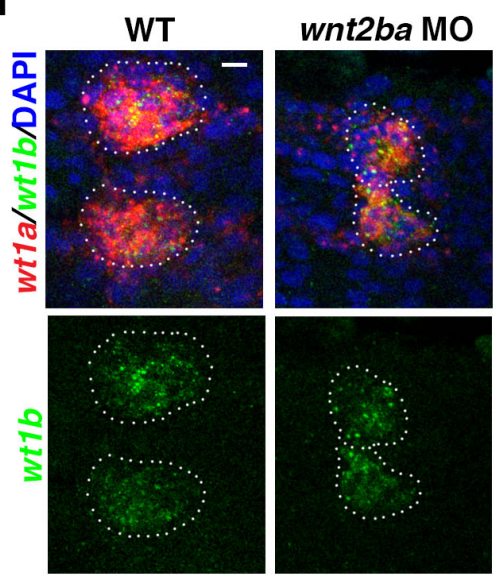

B
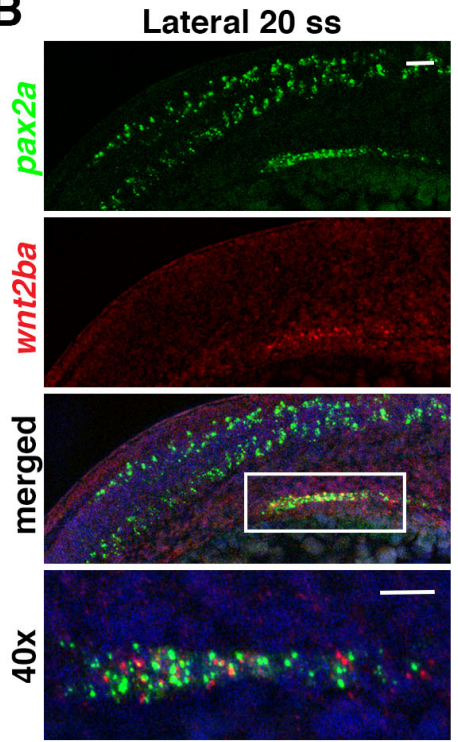

E
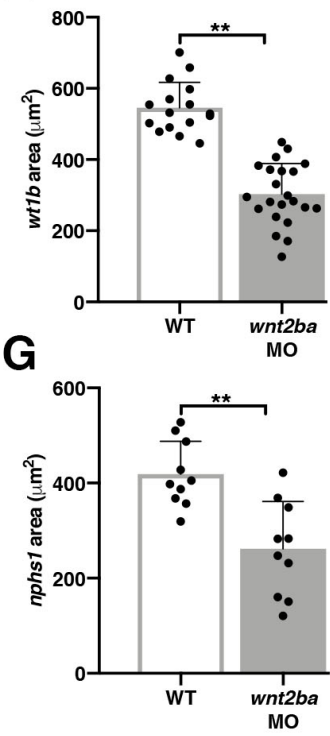

J

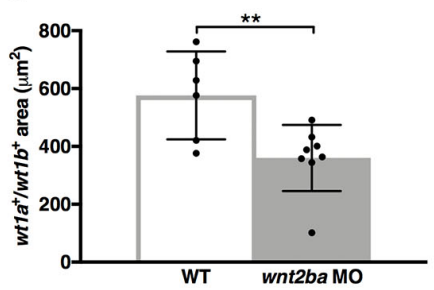

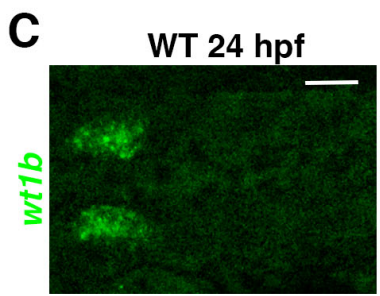
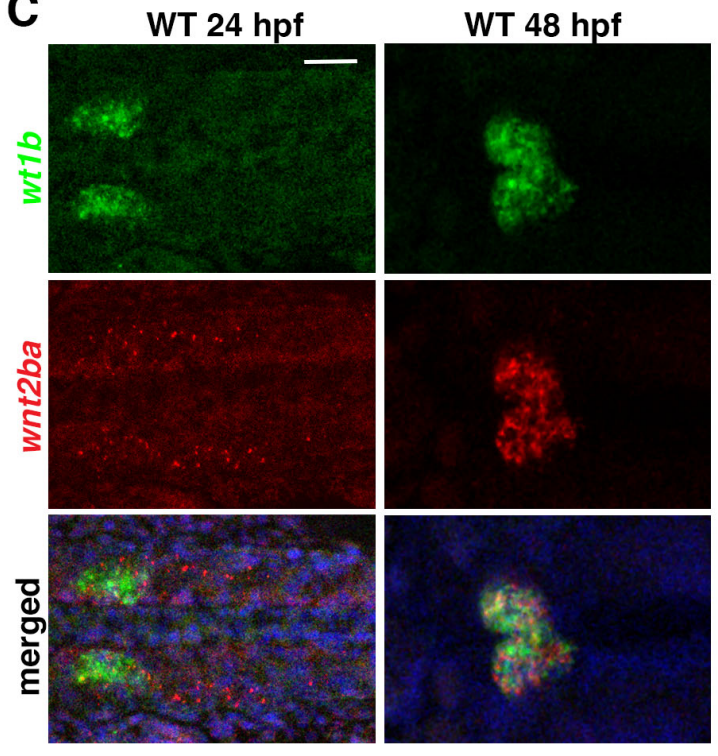

F
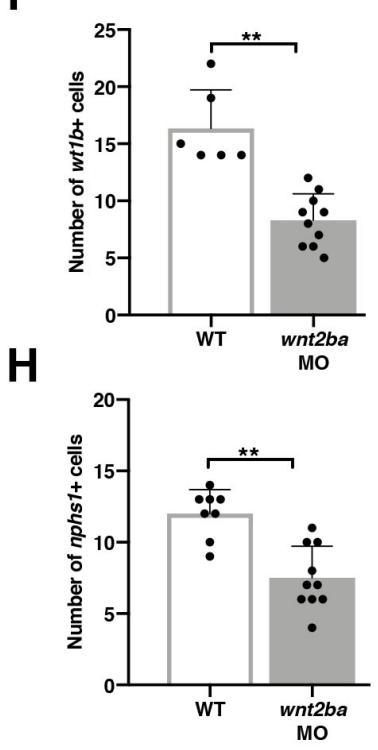
bioRxiv preprint doi: https://doi.org/10.1101/2020.12.21.423845; this version posted December 22, 2020. The copyright holder for this preprint (which was not certified by peer review) is the author/funder, who has granted bioRxiv a license to display the preprint in perpetuity. It is made

\section{Figure 4, Supplement 1} available under aCC-BY-NC-ND 4.0 International license.

A

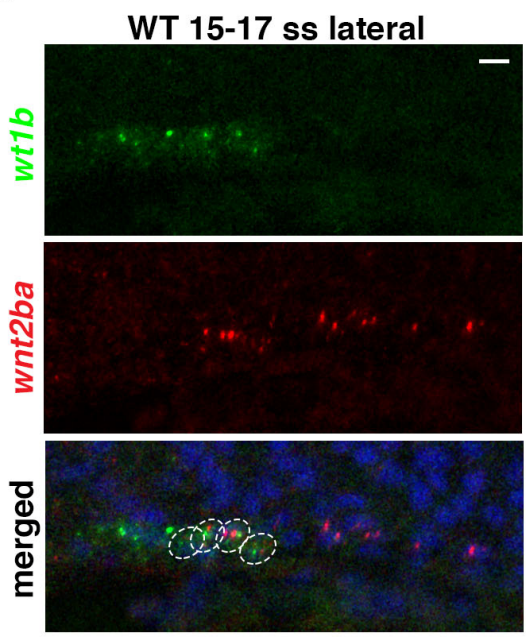

B

WT 22-24 ss lateral

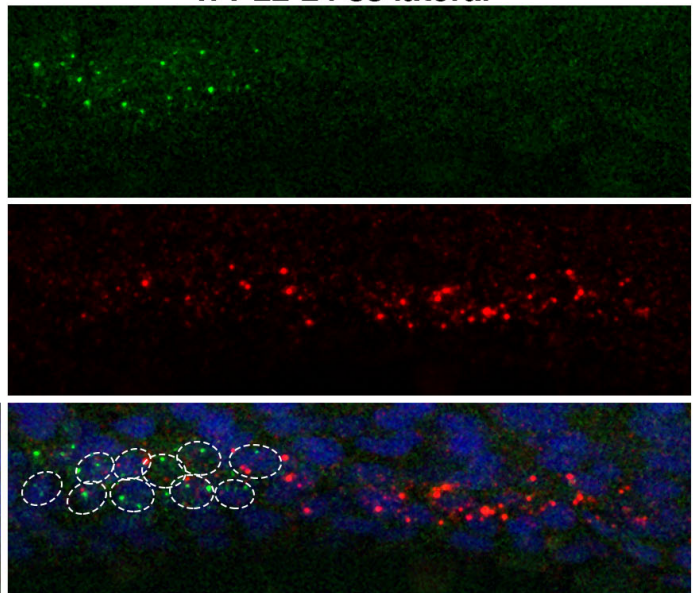

WT 15-17 ss lateral

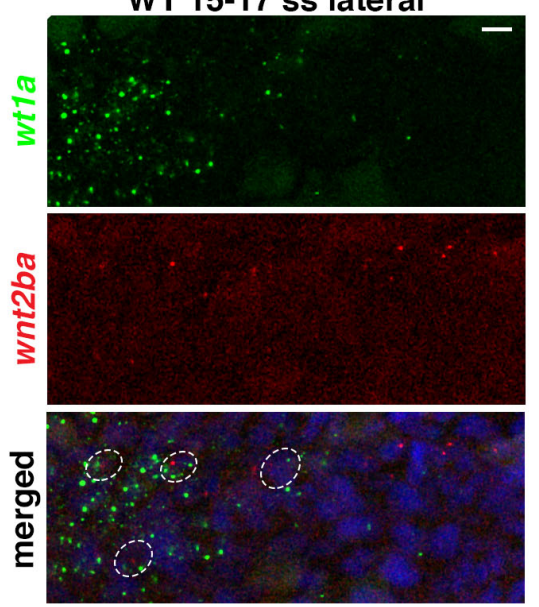


bioRxiv preprint doi: https://doi.org/10.1101/2020.12.21.423845; this version posted December 22, 2020. The copyright holder for this preprint (which was not certified by peer review) is the author/funder, who has granted bioRxiv a license to display the preprint in perpetuity. It is made

\section{Figure 4, Supplement 2} available under aCC-BY-NC-ND 4.0 International license.

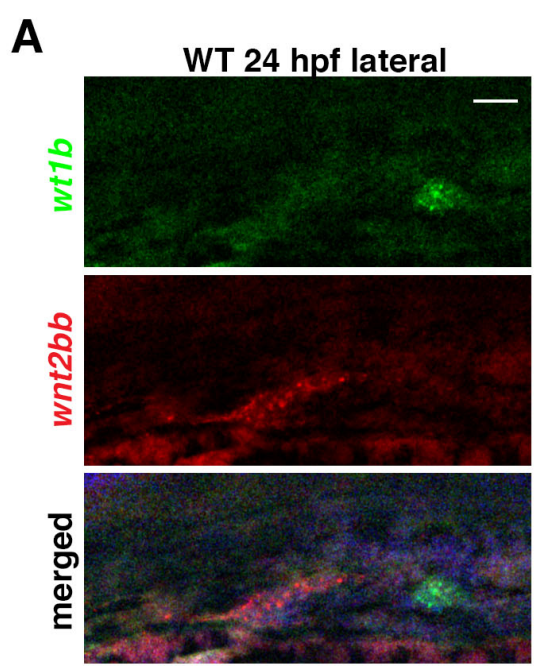


bioRxiv preprint doi: https://doi.org/10.1101/2020.12.21.423845; this version posted December 22, 2020. The copyright holder for this preprint (which was not certified by peer review) is the author/funder, who has granted bioRxiv a license to display the preprint in perpetuity. It is made available under aCC-BY-NC-ND 4.0 International license.

\section{Figure 4 Supplemental 3}

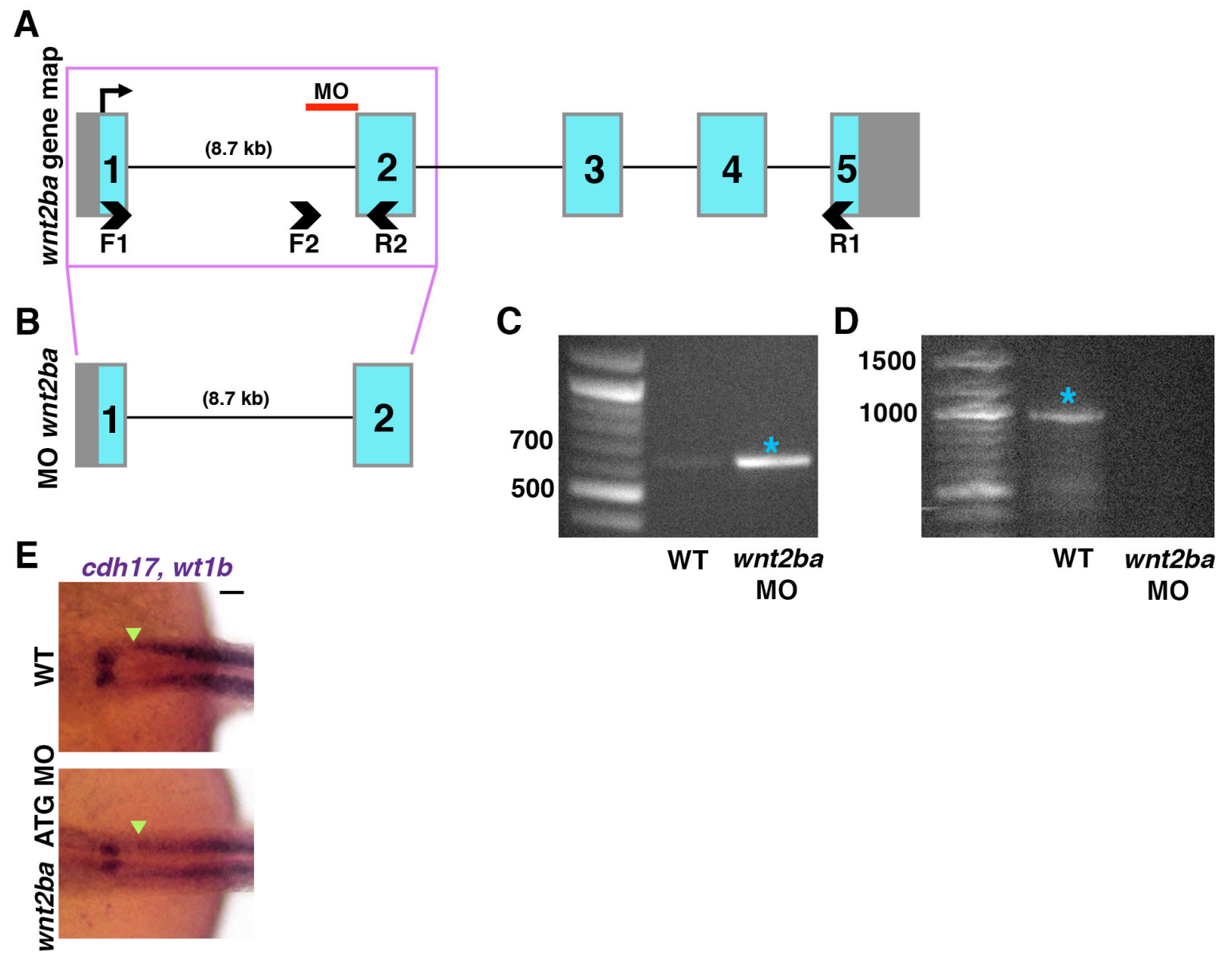


bioRxiv preprint doi: https://doi.org/10.1101/2020.12.21.423845; this version posted December 22, 2020. The copyright holder for this preprint (which was not certified by peer review) is the author/funder, who has granted bioRxiv a license to display the preprint in perpetuity. It is made

Figure 4 Supplement 4

A

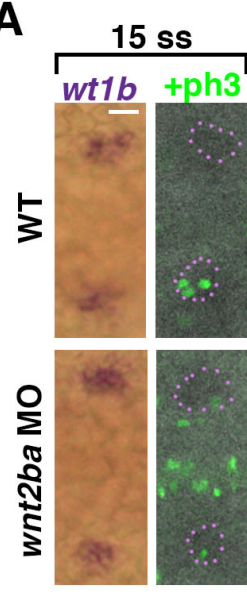

B

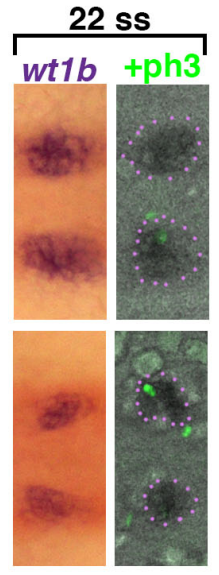
available under aCC-BY-NC-ND 4.0 International license.

D

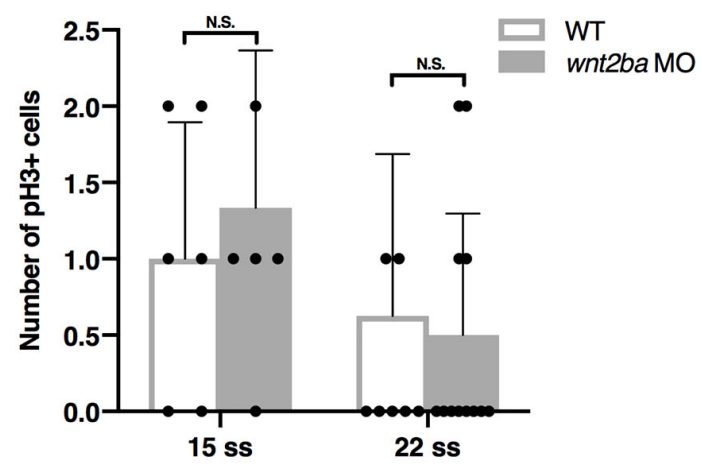

C

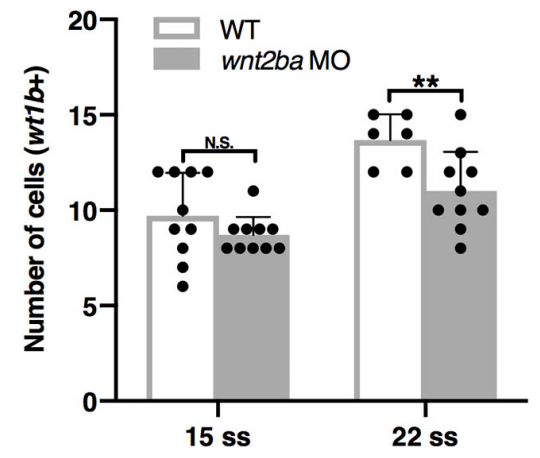


bioRxiv preprint doi: https://doi.org/10.1101/2020.12.21.423845; this version posted December 22, 2020. The copyright holder for this preprint (which was not certified by peer review) is the author/funder, who has granted bioRxiv a license to display the preprint in perpetuity. It is made available under aCC-BY-NC-ND 4.0 International license.

\section{Figure 5}

A
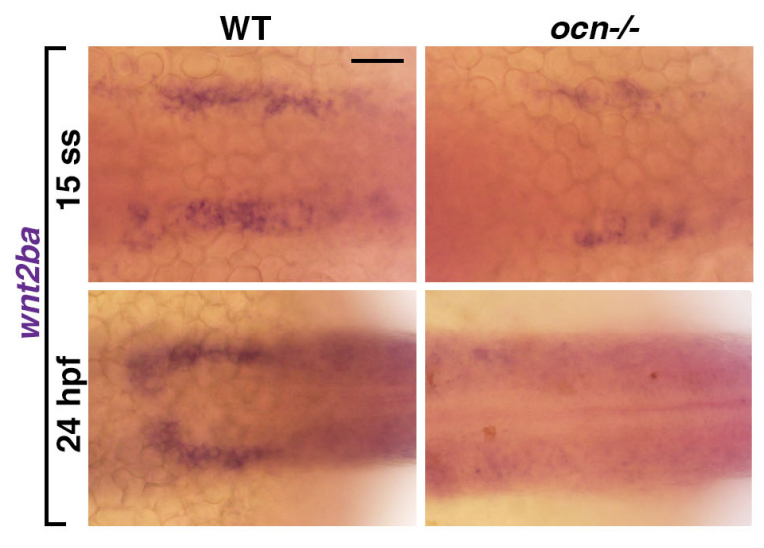

B
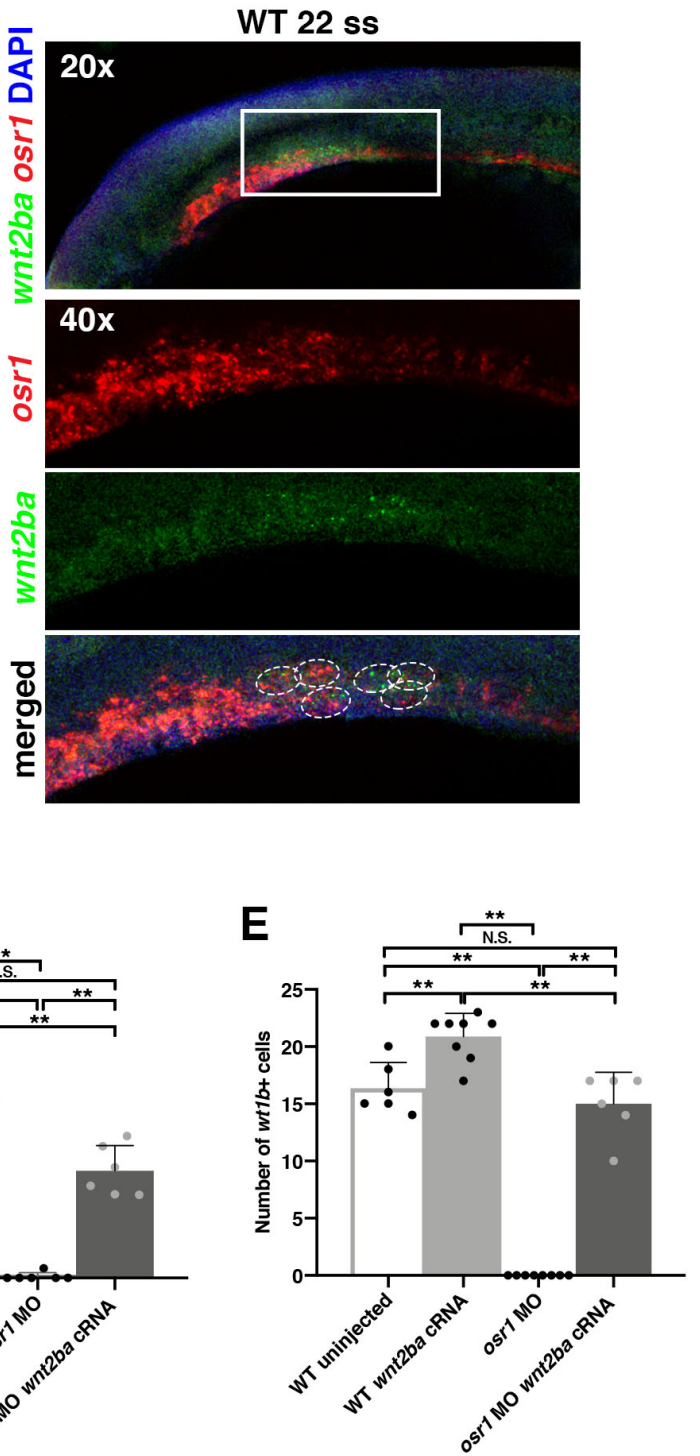

G

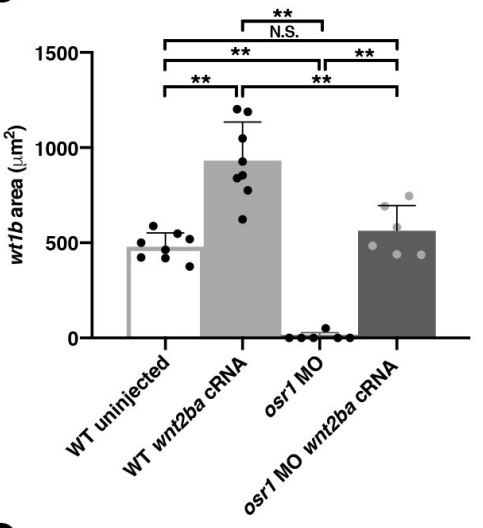

F wT

C

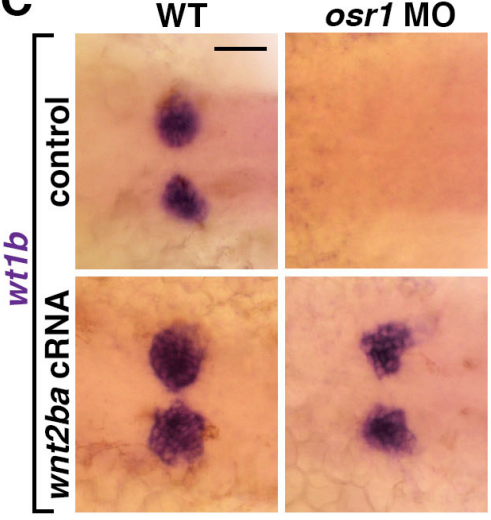

osr1 MO
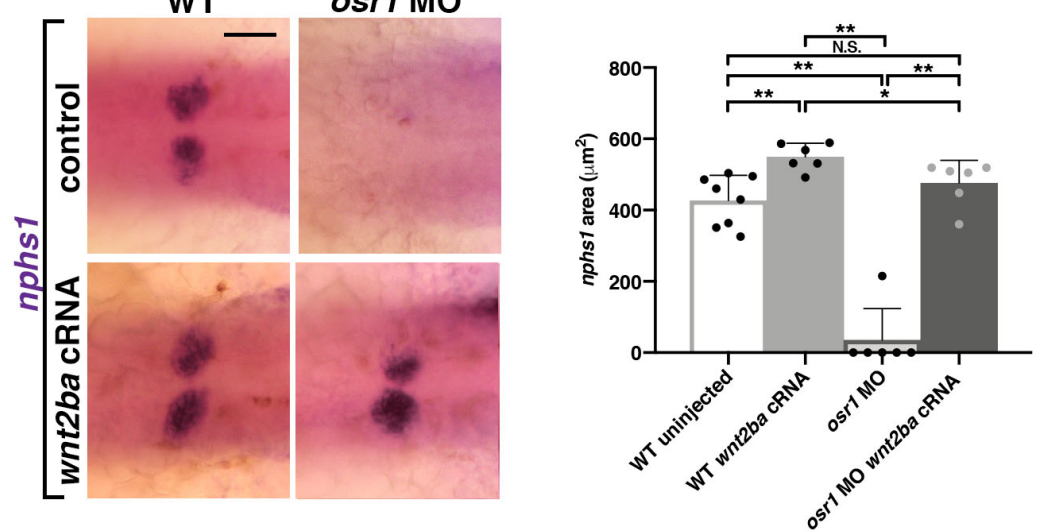

H

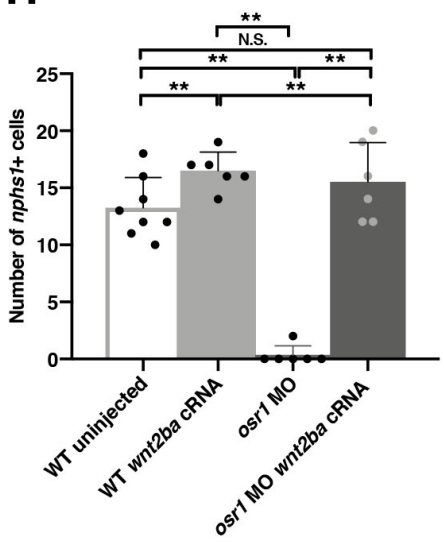


bioRxiv preprint doi: https://doi.org/10.1101/2020.12.21.423845; this version posted December 22, 2020. The copyright holder for this preprint (which was not certified by peer review) is the author/funder, who has granted bioRxiv a license to display the preprint in perpetuity. It is made available under aCC-BY-NC-ND 4.0 International license.

\section{Figure 5, Supplement 1}

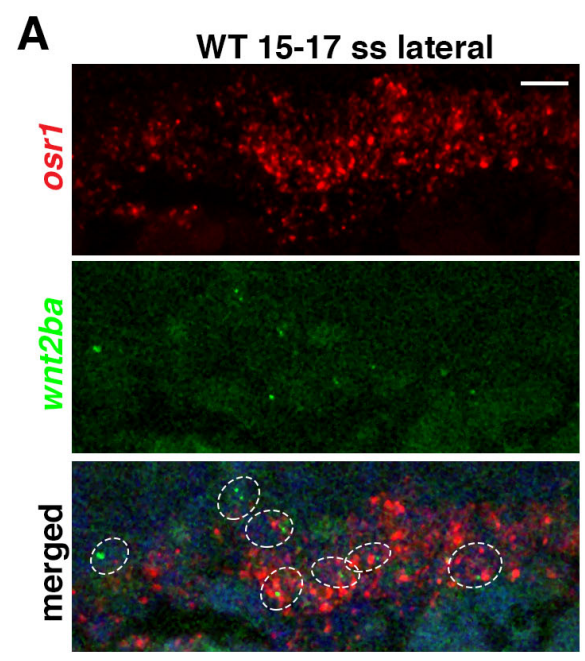




\section{Figure 6}

A

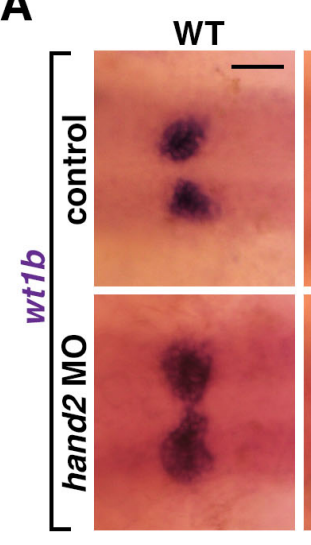

D

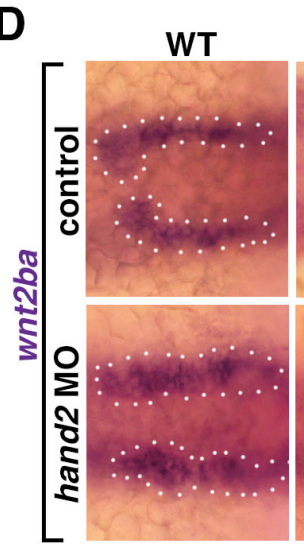

B

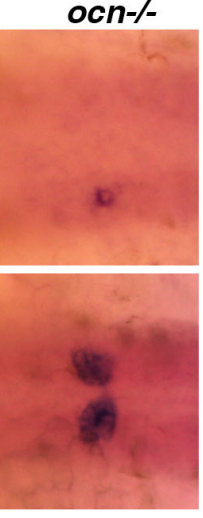

osr1 MO

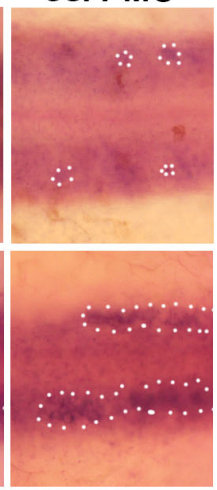

E
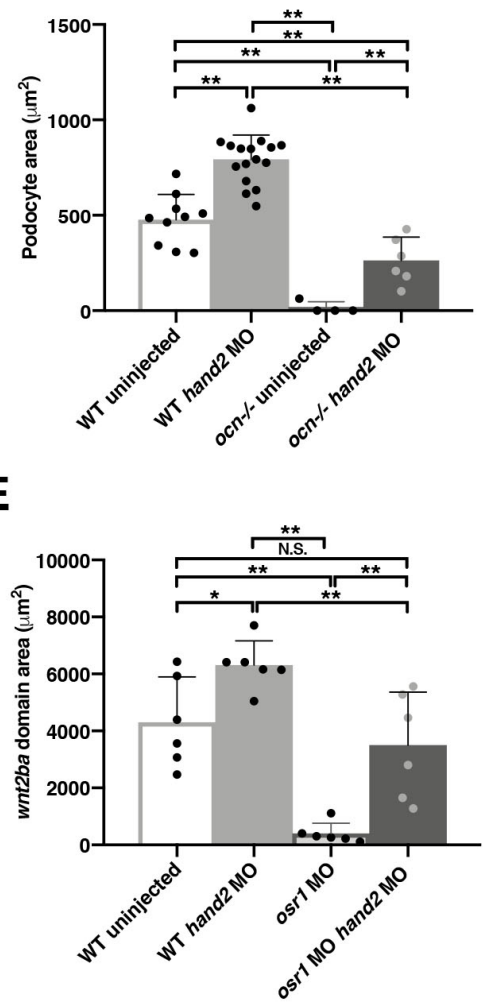

C

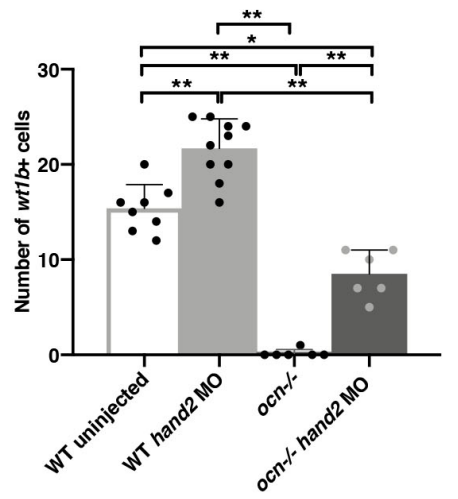

$\mathbf{F}$

5-10 ss

20-28 ss

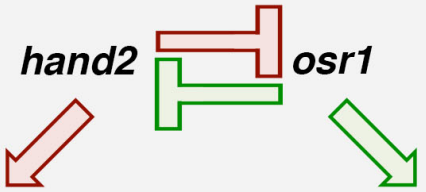

Blood/Vasculature

Progenitors

Kidney

Progenitors wnt2ba

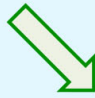

Podocyte program

wt1a

$$
\text { wt1b }
$$

nphs1 
bioRxiv preprint doi: https://doi.org/10.1101/2020.12.21.423845; this version posted December 22, 2020. The copyright holder for this preprint (which was not certified by peer review) is the author/funder, who has granted bioRxiv a license to display the preprint in perpetuity. It is made available under aCC-BY-NC-ND 4.0 International license.

\section{Figure 6, Supplement 1}

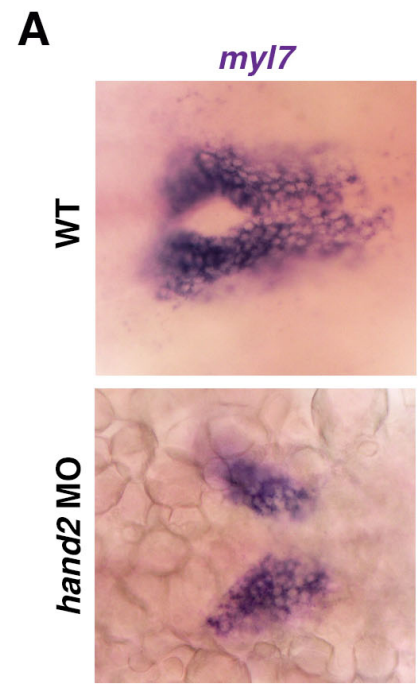

